\title{
The infinitesimal Hopf algebra and the poset of planar forests
}

\author{
L. Foissy
}

Received: 25 February 2008 / Accepted: 4 November 2008 / Published online: 28 November 2008

(C) Springer Science+Business Media, LLC 2008

\begin{abstract}
We introduce an infinitesimal Hopf algebra of planar trees, generalising the construction of the non-commutative Connes-Kreimer Hopf algebra. A nondegenerate pairing and a dual basis are defined, and a combinatorial interpretation of the pairing in terms of orders on the vertices of planar forests is given. Moreover, the coproduct and the pairing can also be described with the help of a partial order on the set of planar forests, making it isomorphic to the Tamari poset. As a corollary, the dual basis can be computed with a Möbius inversion.
\end{abstract}

Keywords Infinitesimal Hopf algebra $\cdot$ Planar tree $\cdot$ Tamari poset

\section{Introduction}

The Connes-Kreimer Hopf algebra of rooted trees (or its dual) is introduced and studied in $[6,10,13,15-17,20,21]$. This commutative, non commutative Hopf algebra is used to treat a problem of Renormalisation in Quantum Field Theory, as explained in $[7,8]$. A non-commutative version of this Hopf algebra is introduced simultaneously in [11] and [14]. This Hopf algebra $\mathcal{H}_{P, R}$, based on planar rooted trees, is neither commutative nor cocommutative, and satisfies a universal property involving a certain coalgebra cohomology [9]. This property is used in [10] to prove that $\mathcal{H}_{P, R}$ is isomorphic to its (graded) dual. In other terms, $\mathcal{H}_{P, R}$ possesses a non-degenerate, symmetric Hopf pairing, and a dual basis of its basis of planar forests. This pairing admits a description in terms of two partial orders on the vertices of the planar forests.

Our aim in the present text is to introduce an infinitesimal version of this Hopf algebra $\mathcal{H}_{P, R}$. The concept of infinitesimal Hopf algebra is introduced in $[3,19]$.

\footnotetext{
L. Foissy ( $₫)$

Laboratoire de Mathématiques, Université de Reims, Moulin de la Housse, BP 1039, 51687 REIMS

Cedex 2, France

e-mail: loic.foissy@univ-reims.fr
} 
Namely, an infinitesimal bialgebra is a space $A$, both an associative, unitary algebra and a coassociative, counitary coalgebra, with the following compatibility:

$$
\Delta(a b)=(a \otimes 1) \Delta(b)+\Delta(a)(1 \otimes b)-a \otimes b .
$$

If it has an antipode, $A$ will be said to be an infinitesimal Hopf algebra. It is proved in [19] that an infinitesimal bialgebra $A$, which is connected as a coalgebra, is isomorphic to $T(\operatorname{Prim}(A))$, with its concatenation product and deconcatenation coproduct: this is the infinitesimal rigidity theorem.

We here construct an infinitesimal coproduct over the algebra $\mathcal{H}$ of planar rooted trees (theorem 9). We use for this the fact that $\mathcal{H}$, given the linear map $B^{+}$of grafting on a common root, is an initial object in a certain category. This infinitesimal coproduct is given by totally admissible cuts (theorem 10), whereas the usual Hopf coproduct is given by admissible cuts. We also give a description of this coproduct in terms of the two partial orders $\geq_{\text {high }}$ and $\geq_{\text {left }}$ on the vertices of a planar forest (proposition 12). We also give a formula for the antipode in terms of left cuts (proposition 15). Using the infinitesimal universal property of $\mathcal{H}$ (theorem 16), we construct a non-degenerate Hopf pairing between $\mathcal{H}$ and $\mathcal{H}^{\text {op,cop }}$ (theorem 19), and a dual basis $\left(f_{F}\right)$ of the basis of forests of $\mathcal{H}$. This pairing $\langle-,-\rangle$ admits a combinatorial interpretation, described in theorem 24 . All these results are infinitesimal versions of the classical Hopf results of [11].

Differences between the infinitesimal and the Hopf case become clear with the observation that the pairing $\langle F, G\rangle$ of two forests $F$ and $G$ is always 0 or 1 in the infinitesimal case. This leads to an interpretation of this pairing in terms of a certain poset, namely the poset of planar forests. A partial order is defined on the set of planar forests with the help of certain transformations of forests (definition 25). This poset $\mathbf{F}$ is isomorphic to the Tamari poset of planar binary trees [23], as it is proved in theorem 31. As a consequence, it has an order-reversing automorphism $m$, corresponding to the vertical symmetry of planar binary trees in the Tamari poset. The pairing $\langle-,-\rangle$ satisfies the following property: for all planar forests $F$ and $G$, $\langle F, G\rangle=1$ if, and only if, $F \geq m(G)$ in $\mathbf{F}$. As a consequence, the dual basis $\left(f_{F}\right)$ is given by a Möbius inversion (corollary 39). Moreover, the product of two elements of the dual basis admits also a description using suborders of $\mathbf{F}$ (corollary 29). We shall show in another text that this dual basis can be iteratively computed with the help of two operads of planar forests. For the sake of simplicity, we restrict ourselves here to planar rooted trees with no decorations, but there exists versions of all these results for planar decorated rooted trees, and these versions are proved similarly.

Note that we introduced in [12] a $q$-deformation $\mathcal{H}_{q}$ of $\mathcal{H}_{P, R}$. In the special case $q=0$, we obtain the infinitesimal Hopf algebra of planar rooted trees of the present paper. Moreover, if $q \neq 0, \mathcal{H}_{q}$ has a non-degenerate pairing also described in [12]. For $q=0$, this pairing becomes degenerate and is not the pairing described here.

This paper is organised as follows: the first section is devoted to recalls and complements about infinitesimal Hopf algebras. In particular, it is proved that for any infinitesimal Hopf algebra $A, \operatorname{Ker}(\varepsilon)=\operatorname{Prim}(A) \oplus \operatorname{Ker}(\varepsilon)^{2}$, and the projector on $\operatorname{Prim}(A)$ in this direct sum is the antipode, recovering in this way the rigidity theorem of [19]. The infinitesimal Hopf algebra of planar rooted trees $\mathcal{H}$ is introduced in the second section. We construct its infinitesimal coproduct and give its description 
in terms of totally admissible cuts and biideals, before a formula for the antipode. We prove a universal property of $\mathcal{H}$ and use it to construct a Hopf pairing between $\mathcal{H}$ and $\mathcal{H}^{o p, c o p}$. The combinatorial description of this pairing is then given. The last section deals with the poset of forests $\mathbf{F}$ and its applications. We prove that this poset is isomorphic to the Tamari poset and describe an order-reversing automorphism of $\mathbf{F}$. The link between the pairing of $\mathcal{H}$ and the order on $\mathbf{F}$ is then given.

Notation. We denote by $K$ a commutative field, of any characteristic. Every algebra, coalgebra, etc, will be taken over $K$.

\section{Recalls on infinitesimal Hopf algebras}

We refer to $[1,24]$ for the classical results and definitions about coalgebras, bialgebras, Hopf algebras.

\subsection{Infinitesimal Hopf algebras}

Definition 1 (See [3, 19]).

1. An infinitesimal bialgebra is an associative, unitary algebra $A$, together with a coassociative, counitary coproduct, satisfying the following compatibility: for all $a, b \in A$,

$$
\Delta(a b)=\Delta(a)(1 \otimes b)+(a \otimes 1) \Delta(b)-a \otimes b .
$$

2. Let $A$ be an infinitesimal bialgebra. If $I d_{A}$ has an inverse $S$ in the associative convolution algebra $(\mathcal{L}(A), \star)$, we shall say that $A$ is an infinitesimal Hopf algebra, and $S$ will be called the antipode of $A$.

\section{Remarks.}

1. This is not the same definition as used by Aguiar in [2].

2. Infinitesimal bialgebras are bimonoids in a certain braided monoidal category (in which the braiding is not an isomorphism), as this appears in remark A.3.2 of [3]. As a consequence, several results of this section, for example proposition 2-3, are special cases of general results for bimonoids in braided monoidal categories, see [25].

3. Let $A$ be an infinitesimal bialgebra and let $M$ be the kernel of its counit. We shall prove in proposition 2 that $M$ is an ideal. Moreover, $M$ is given a coassociative, non counitary coproduct $\tilde{\Delta}$ defined by:

$$
\tilde{\Delta}:\left\{\begin{aligned}
M & \longrightarrow M \otimes M \\
x & \longrightarrow \Delta(x)-x \otimes 1-1 \otimes x .
\end{aligned}\right.
$$

The compatibility between $\tilde{\Delta}$ and the product is given by the non (co)unital infinitesimal compatibility:

$$
\tilde{\Delta}(a b)=(a \otimes 1) \tilde{\Delta}(b)+\tilde{\Delta}(a)(1 \otimes b)+a \otimes b .
$$


4. By induction, if $x_{1}, \ldots, x_{n} \in M$ :

$$
\begin{aligned}
\tilde{\Delta}\left(x_{1} \ldots x_{n}\right)= & \sum_{i=1}^{n-1} x_{1} \ldots x_{i} \otimes x_{i+1} \ldots x_{n} \\
& +\sum_{i=1}^{n}\left(x_{1} \ldots x_{i-1} \otimes 1\right) \tilde{\Delta}\left(x_{i}\right)\left(1 \otimes x_{i+1} \ldots x_{n}\right) .
\end{aligned}
$$

5. In particular, if $x_{1}, \ldots, x_{n}$ are primitive elements of $A$ :

$$
\Delta\left(x_{1} \ldots x_{n}\right)=\sum_{i=0}^{n} x_{1} \ldots x_{i} \otimes x_{i+1} \ldots x_{n} .
$$

Notations. Let $A$ be an infinitesimal bialgebra. For all $x \in A$, we denote $\Delta(x)=$ $x^{(1)} \otimes x^{(2)}$. Moreover, if $\varepsilon(x)=0$, we denote $\tilde{\Delta}(x)=x^{\prime} \otimes x^{\prime \prime}$.

\section{Examples.}

1. Let $V$ be a vector space. The tensor algebra $T(V)$ is given a structure of infinitesimal Hopf algebra with the coproduct $\Delta$ defined, for $v_{1}, \ldots, v_{n} \in V$, by:

$$
\Delta\left(v_{1} \ldots v_{n}\right)=\sum_{i=0}^{n} v_{1} \ldots v_{i} \otimes v_{i+1} \ldots v_{n} .
$$

It is proved in [19] that any connected (as a coalgebra) infinitesimal bialgebra $A$ is isomorphic to $T(\operatorname{Prim}(A))$.

2. If $A$ is an infinitesimal Hopf algebra, then $A^{o p, c o p}$ also is, with the same antipode. Note that $A^{o p}$ and $A^{c o p}$ are not infinitesimal bialgebras, as the compatibility (1) is no more satisfied.

3. If $A$ is a graded infinitesimal Hopf algebra, such that its homogeneous components are finite-dimensional, then its graded dual $A^{*}$ also is.

\section{Proposition 2 Let $A$ be an infinitesimal bialgebra.}

1. Then $\Delta(1)=1 \otimes 1$. In other terms, the unit map $v$ is a coalgebra morphism:

$$
v:\left\{\begin{aligned}
K & \longrightarrow A \\
1 & \longrightarrow
\end{aligned}\right.
$$

2. The counit $\varepsilon$ is an algebra morphism.

3. If, moreover, $A$ is an infinitesimal Hopf algebra, then $S(1)=1$ and $\varepsilon \circ S=\varepsilon$.

\section{Proof.}

1. For $a=b=1$, relation (1) becomes $\Delta(1)=\Delta(1)+\Delta(1)-1 \otimes 1$. So $\Delta(1)=$ $1 \otimes 1$. As a consequence, $\varepsilon(1)=1$. Moreover, if $A$ has an antipode, $S(1)=1$. 
2. For $a, b \in A$ :

$$
\begin{aligned}
(\varepsilon \otimes \varepsilon) \circ \Delta(a b) & =\varepsilon\left(a^{(1)}\right) \varepsilon\left(a^{(2)} b\right)+\varepsilon\left(a b^{(1)}\right) \varepsilon\left(b^{(2)}\right)-\varepsilon(a) \varepsilon(b) \\
& =\varepsilon(a b)+\varepsilon(a b)-\varepsilon(a) \varepsilon(b) \\
& =\varepsilon(a b) .
\end{aligned}
$$

So $\varepsilon(a b)=\varepsilon(a) \varepsilon(b)$.

3. For all $a \in A$ :

$$
\varepsilon(a)=\varepsilon(\varepsilon(a) 1)=\varepsilon \circ m \circ(S \otimes I d) \circ \Delta(a)=\varepsilon\left(S\left(a^{(1)}\right)\right) \varepsilon\left(a^{(2)}\right)=\varepsilon(S(a)) .
$$

So $\varepsilon \circ S=\varepsilon$.

\section{Lemma 3}

1. Let $A, B$ be two augmented algebras, with respective augmentations denoted by $\varepsilon_{A}: A \longrightarrow K$ and $\varepsilon_{B}: B \longrightarrow K$. Then $A \otimes B$ is an associative, unitary algebra, with product ${ }_{\varepsilon_{A, B}}$ given for all $a_{1}, a_{2} \in A, b_{1}, b_{2} \in B$, by:

$\left(a_{1} \otimes b_{1}\right) \cdot_{\varepsilon_{A, B}}\left(a_{2} \otimes b_{2}\right)=\varepsilon\left(a_{2}\right) a_{1} \otimes b_{1} b_{2}+\varepsilon\left(b_{1}\right) a_{1} a_{2} \otimes b_{2}-\varepsilon_{A}\left(a_{2}\right) \varepsilon_{B}\left(b_{1}\right) a_{1} \otimes b_{2}$.

The unit is $1_{A} \otimes 1_{B}$.

2. Let $A, B$ be two pointed coalgebras, with group-like elements $1_{A}$ and $1_{B}$. Then $A \otimes B$ is a coassociative, counitary coalgebra, with coproduct $\Delta_{1_{A, B}}$ given by:

$\Delta_{1_{A, B}}(a \otimes b)=a \otimes b^{(1)} \otimes 1_{A} \otimes b^{(2)}+a^{(1)} \otimes 1_{B} \otimes a^{(2)} \otimes b-a \otimes 1_{B} \otimes 1_{A} \otimes b$.

The counit is $\varepsilon_{A} \otimes \varepsilon_{B}$.

Proof. Direct computations.

\section{Remarks.}

1. Lemma 3-1 is proposition B.4 of [3].

2. When the augmented algebras $A$ and $B$ are equal, we shall simply denote $\varepsilon_{A}=$ $\varepsilon_{B}=\varepsilon$, and $\Delta_{\varepsilon_{A, B}}=\Delta_{\varepsilon}$. When the pointed coalgebras $A$ and $B$ are equal, we shall denote $1_{A}=1_{B}=1$ and $\Delta_{1_{A, B}}=\Delta_{1}$.

3. If $A$ is an infinitesimal bialgebra, then compatibility (1) means that the coproduct $\Delta:(A, \cdot) \longrightarrow\left(A \otimes A,{ }_{\varepsilon}\right)$, where $\varepsilon$ is the counit of $A$, is a morphism of algebras. Indeed, for all $a, b \in A$ :

$$
\begin{aligned}
\Delta(a){ }_{\varepsilon} \Delta(b)= & \varepsilon\left(b^{(1)}\right) a^{(1)} \otimes a^{(2)} b^{(2)}+\varepsilon\left(a^{(2)}\right) a^{(1)} b^{(1)} \otimes b^{(2)} \\
& -\varepsilon\left(a^{(2)}\right) \varepsilon\left(b^{(1)}\right) a^{(1)} \otimes b^{(2)} \\
= & a^{(1)} \otimes a^{(2)} b+a b^{(1)} \otimes b^{(2)}-a \otimes b .
\end{aligned}
$$

Dually, it also means that the product $m:\left(A \otimes A, \Delta_{1}\right) \longrightarrow(A, \Delta)$ is a morphism of coalgebras. 


\subsection{Antipode of an infinitesimal Hopf algebra}

Lemma 4 Let $A$ be an infinitesimal Hopf algebra.

1. For all $a, b \in A, S(a b)=\varepsilon(a) S(b)+\varepsilon(b) S(a)-\varepsilon(a) \varepsilon(b) 1$. In particular, for all $a, b \in A$, such that $\varepsilon(a)=\varepsilon(b)=0, S(a b)=0$.

2. For all $a \in A, \Delta(S(a))=S(a) \otimes 1+1 \otimes S(a)-\varepsilon(a) 1 \otimes 1$. In particular, for all $a \in A$, such that $\varepsilon(a)=0, S(a)$ is primitive.

\section{Proof.}

1. Let us consider the convolution algebra $\mathcal{L}(A \otimes A, A)$, where $A \otimes A$ is given the coproduct of lemma 3. For all $a, b \in A$ :

$$
\begin{aligned}
&((S \circ m) \star m)(a \otimes b) \\
&= m \circ((S \circ m) \otimes m) \circ \Delta_{1}(a \otimes b) \\
&= m \circ((S \circ m) \otimes m)\left(a^{(1)} \otimes 1 \otimes a^{(2)} \otimes b+a \otimes b^{(1)} \otimes 1 \otimes b^{(2)}\right. \\
&-a \otimes 1 \otimes 1 \otimes b) \\
&= S\left(a^{(1)}\right) a^{(2)} b+S\left(a b^{(1)}\right) b^{(2)}-S(a) b \\
&= m \circ(S \otimes I d) \circ \Delta(a b) \\
&= \varepsilon(a b) 1 \\
&= \varepsilon(a) \varepsilon(b) 1 .
\end{aligned}
$$

So $S \circ m$ is a left inverse of $m$.

Let $T: A \otimes A \longrightarrow A$ defined by $T(a \otimes b)=\varepsilon(a) S(b)+\varepsilon(b) S(a)-\varepsilon(a) \varepsilon(b) 1$. Let us compute $m \star T$ in $\mathcal{L}(A \otimes A, A)$ :

$$
\begin{aligned}
&(m \star T)(a \otimes b) \\
&= m \circ(m \otimes T) \circ \Delta_{1}(a \otimes b) \\
&= m \circ(m \otimes T)\left(a^{(1)} \otimes 1 \otimes a^{(2)} \otimes b+a \otimes b^{(1)} \otimes 1 \otimes b^{(2)}-a \otimes 1 \otimes 1 \otimes b\right) \\
&= a^{(1)}\left(\varepsilon\left(a^{(2)}\right) S(b)+\varepsilon(b) S\left(a^{(2)}\right)-\varepsilon\left(a^{(2)}\right) \varepsilon(b) 1\right) \\
&+a b^{(1)}\left(S\left(b^{(2)}\right)+\varepsilon\left(b^{(2)}\right) 1-\varepsilon\left(b^{(2)}\right) 1\right)-a(S(b)+\varepsilon(b) 1-\varepsilon(b) 1) \\
&= a S(b)+\varepsilon(a) \varepsilon(b) 1-\varepsilon(b) a+\varepsilon(b) a-a S(b) \\
&= \varepsilon(a) \varepsilon(b) 1 .
\end{aligned}
$$

So $T$ is a right inverse of $m$. As the convolution product is associative, $S \circ m=T$.

2. Let us consider the convolution algebra $\mathcal{L}(A, A \otimes A)$, where $A \otimes A$ is given the product of lemma 3. For all $a \in A$ : 


$$
\begin{aligned}
&((\Delta \circ S) \star \Delta)(a) \\
&= m_{\varepsilon} \circ((\Delta \circ S) \otimes \Delta) \circ \Delta(a) \\
&= m_{\varepsilon}\left(S\left(a^{(1)}\right)^{(1)} \otimes S\left(a^{(1)}\right)^{(2)} \otimes a^{(2)} \otimes a^{(3)}\right) \\
&= \varepsilon\left(S\left(a^{(1)}\right)^{(2)}\right) S\left(a^{(1)}\right)^{(1)} a^{(2)} \otimes a^{(3)}+\varepsilon\left(a^{(2)}\right) S\left(a^{(1)}\right)^{(1)} \otimes S\left(a^{(1)}\right)^{(2)} a^{(3)} \\
&-\varepsilon\left(S\left(a^{(1)}\right)^{(2)}\right) \varepsilon\left(a^{(2)}\right) S\left(a^{(1)}\right)^{(1)} \otimes a^{(3)} \\
&= S\left(a^{(1)}\right) a^{(2)} \otimes a^{(3)}+S\left(a^{(1)}\right)^{(1)} \otimes S\left(a^{(1)}\right)^{(2)} a^{(2)}-S\left(a^{(1)}\right) \otimes a^{(2)} \\
&= \Delta\left(S\left(a^{(1)}\right) a^{(2)}\right) \\
&= \varepsilon(a) \Delta(1) \\
&= \varepsilon(a) 1 \otimes 1 .
\end{aligned}
$$

So $\Delta \circ S$ is a left inverse of $\Delta$.

Let $T: A \longrightarrow A \otimes A$ defined by $T(a)=S(a) \otimes 1+1 \otimes S(a)-\varepsilon(a) 1 \otimes 1$. Let us compute $\Delta \star T$ in $\mathcal{L}(A, A \otimes A)$ :

$$
\begin{aligned}
&(\Delta \star T)(a) \\
&= m_{\varepsilon} \circ(\Delta \otimes T) \circ \Delta(a) \\
&= m_{\varepsilon}\left(a^{(1)} \otimes a^{(2)} \otimes 1 \otimes S\left(a^{(3)}\right)+a^{(1)} \otimes a^{(2)} \otimes S\left(a^{(3)}\right) \otimes 1\right. \\
&\left.-\varepsilon\left(a^{(3)}\right) a^{(1)} \otimes a^{(2)} \otimes 1 \otimes 1\right) \\
&= a^{(1)} \otimes a^{(2)} S\left(a^{(3)}\right)+\varepsilon\left(a^{(2)}\right) a^{(1)} \otimes S\left(a^{(3)}\right)-\varepsilon\left(a^{(2)}\right) a^{(1)} \otimes S\left(a^{(3)}\right) \\
&+\varepsilon\left(S\left(a^{(3)}\right)\right) a^{(1)} \otimes a^{(2)}+\varepsilon\left(a^{(2)}\right) a^{(1)} S\left(a^{(3)}\right) \otimes 1 \\
&-\varepsilon\left(a^{(2)}\right) \varepsilon\left(S\left(a^{(3)}\right)\right) a^{(1)} \otimes 1 \\
&-a^{(1)} \otimes a^{(2)}-\varepsilon\left(a^{(2)}\right) a^{(1)} \otimes 1+\varepsilon\left(a^{(2)}\right) a^{(1)} \otimes 1 \\
&= a \otimes 1+a^{(1)} \otimes a^{(2)}+\varepsilon(a) 1 \otimes 1-a \otimes 1-a^{(1)} \otimes a^{(2)}-a \otimes 1+a \otimes 1 \\
&= \varepsilon(a) 1 \otimes 1 .
\end{aligned}
$$

So $T$ is a right inverse of $\Delta$. As the convolution product is associative, $\Delta \circ S=T$.

Corollary 5 Let $A$ be an infinitesimal Hopf algebra. Then $\operatorname{Ker}(\varepsilon)=\operatorname{Prim}(A) \oplus$ $\operatorname{Ker}(\varepsilon)^{2}$. The projection on $\operatorname{Prim}(A)$ in this direct sum is $-S$.

Proof. Let $a \in \operatorname{Ker}(\varepsilon)$. Then $\Delta(a)=a \otimes 1+1 \otimes a+a^{\prime} \otimes a^{\prime \prime}$, with $a^{\prime} \otimes a^{\prime \prime} \in \operatorname{Ker}(\varepsilon) \otimes$ $\operatorname{Ker}(\varepsilon)$. Moreover:

$$
0=\varepsilon(a) 1=m \circ(S \otimes I d) \circ \Delta(a)=S(a)+a+S\left(a^{\prime}\right) a^{\prime \prime},
$$

so $a=-S(a)-S\left(a^{\prime}\right) a^{\prime \prime}$. By lemma 4, $-S(a) \in \operatorname{Prim}(A)$ and $S\left(a^{\prime}\right) a^{\prime \prime} \in S(\operatorname{Ker}(\varepsilon)) \times$ $\operatorname{Ker}(\varepsilon)^{2} \subseteq \operatorname{Ker}(\varepsilon)^{2}$, so $\operatorname{Ker}(\varepsilon)=\operatorname{Prim}(A)+\operatorname{Ker}(\varepsilon)^{2}$. If $a \in \operatorname{Prim}(A)$, then 
$a^{\prime} \otimes a^{\prime \prime}=0$, so $-S(a)=a$. Moreover, $S\left(\operatorname{Ker}(\varepsilon)^{2}\right)=(0)$, so $\operatorname{Ker}(\varepsilon)=\operatorname{Prim}(A) \oplus$ $\operatorname{Ker}(\varepsilon)^{2}$ and the projector on $\operatorname{Prim}(A)$ in this direct sum is $-S$.

\section{Remarks.}

1. This result implies the rigidity theorem of [19].

2. It is also possible to prove lemma 4 using braided Hopf algebras.

\section{Infinitesimal Hopf algebra of planar trees}

2.1 Algebra of planar trees and universal property

\section{Definition 6}

1. The set of planar rooted trees will be denoted by $\mathbf{T}$ (see $[11,14]$ ).

2. The algebra $\mathcal{H}$ is the free associative algebra generated by $\mathbf{T}$. The monomials of $\mathcal{H}$ will be called planar forests. The set of planar forests will be denoted by $\mathbf{F}$. The weight of an element $F \in \mathbf{F}$ is the number of its vertices.

\section{Examples.}

1. Planar rooted trees of weight $\leq 5$ :

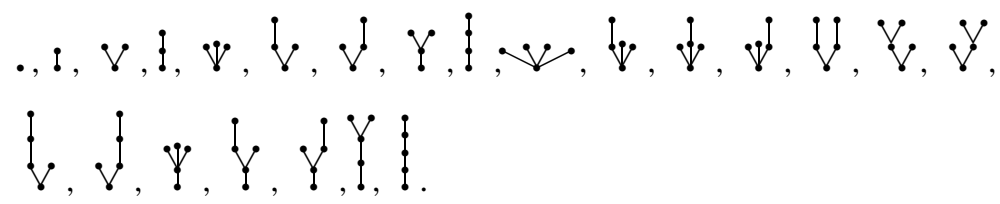

2. Planar rooted forests of weight $\leq 4$ :

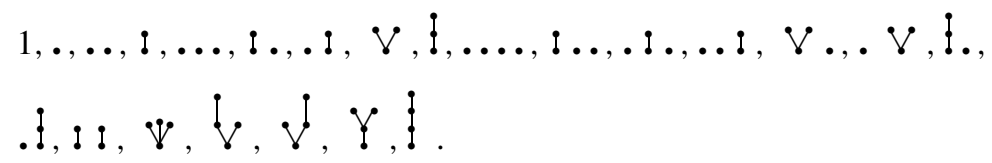

We define the operator $B^{+}: \mathcal{H} \longrightarrow \mathcal{H}$, which associates, to a forest $F \in \mathbf{F}$, the tree obtained by grafting the roots of the trees of $F$ on a common root. For example, $B^{+}(\dot{\bullet})=\dot{V}$, and $B^{+}(.:)=\mathcal{V}$. It is shown in [20] that $\left(\mathcal{H}, B^{+}\right)$is an initial object in the category of couples $(A, L)$, where $A$ is an algebra, and $L: A \longrightarrow A$ any linear operator. More explicitly:

Theorem 7 (Universal property of $\mathcal{H}$ ) Let $A$ be any algebra and let $L: A \longrightarrow A$ be a linear map. Then there exists a unique algebra morphism $\phi: \mathcal{H} \longrightarrow A$, such that $\phi \circ B^{+}=L \circ \phi$. 
Remark. Note that $\phi$ is inductively defined in the following way: for all trees $t_{1}, \ldots, t_{n} \in \mathbf{T}$,

$$
\left\{\begin{aligned}
\phi(1) & =1 \\
\phi\left(t_{1} \ldots t_{n}\right) & =\phi\left(t_{1}\right) \ldots \phi\left(t_{n}\right) \\
\phi\left(B^{+}\left(t_{1} \ldots t_{n}\right)\right) & =L\left(\phi\left(t_{1}\right) \ldots \phi\left(t_{n}\right)\right) .
\end{aligned}\right.
$$

The end of this section is devoted to the introduction of several combinatorial concepts, which will be useful for the sequel.

Definition 8 Let $F \in \mathbf{F}$. An admissible cut is a non empty cut of certain edges and trees of $F$, such that each path in a non-cut tree of $F$ meets at most one cut edge (see $[6,11])$. The set of admissible cuts of $F$ will be denoted by $\operatorname{Adm}(F)$. If $c$ is an admissible cut of $F$, the forest of the vertices which are over the cuts of $c$ will be denoted by $P^{c}(t)$ (branch of the cut $c$ ), and the remaining forest will be denoted by $R^{c}(t)$ (trunk of the cut).

We now recall several order relations on the set of the vertices of a planar forest, see [11] for more details. Let $F=t_{1} \ldots t_{n} \in \mathbf{F}-\{1\}$ and let $s, s^{\prime}$ be two vertices of $F$.

1. We shall say that $s \geq$ high $s^{\prime}$ if there exists a path from $s^{\prime}$ to $s$ in $F$, the edges of $F$ being oriented from the roots to the leaves. Note that $\geq_{h i g h}$ is a partial order, whose Hasse graph is the forest $F$.

2. If $s$ and $s^{\prime}$ are not comparable for $\geq_{h i g h}$, we shall say that $s \geq_{\text {left }} s^{\prime}$ if one of these assertions is satisfied:

(a) $s$ is a vertex of $t_{i}$ and $s^{\prime}$ is a vertex of $t_{j}$, with $i<j$.

(b) $s$ and $s^{\prime}$ are vertices of the same $t_{i}$, and $s \geq_{\text {left }} s^{\prime}$ in the forest obtained from $t_{i}$ by deleting its root.

This defines the partial order $\geq_{\text {left }}$ for all forests $F$, by induction on the the weight.

3. We shall say that $s \geq_{h, l} s^{\prime}$ if $s \geq_{h i g h} s^{\prime}$ or $s \geq_{\text {left }} s^{\prime}$. This defines a total order on the vertices of $F$.

Example. Let $t=\grave{V}$. We index its vertices in the following way: ${ }^{2} \vee_{4}{ }^{3}$. The following arrays give the order relations $\geq_{h i g h}$ and $\geq_{\text {left }}$ for the vertices of $t$. A symbol $\times$ means that the vertices are not comparable for the order.

\begin{tabular}{|c|c|c|c|c|}
\hline$x \backslash y$ & $s_{1}$ & $s_{2}$ & $s_{3}$ & $s_{4}$ \\
\hline$s_{1}$ & $=$ & $\geq_{h i g h}$ & $\times$ & $\geq_{\text {high }}$ \\
\hline$s_{2}$ & $\leq_{\text {high }}$ & $=$ & $\times$ & $\geq_{\text {high }}$ \\
\hline$s_{3}$ & $\times$ & $\times$ & $=$ & $\geq_{\text {high }}$ \\
\hline$s_{4}$ & $\leq_{\text {high }}$ & $\leq_{\text {high }}$ & $\leq_{\text {high }}$ & $=$ \\
\hline
\end{tabular}

\begin{tabular}{|c|c|c|c|c|}
\hline$x \backslash y$ & $s_{1}$ & $s_{2}$ & $s_{3}$ & $s_{4}$ \\
\hline$s_{1}$ & $=$ & $\times$ & $\geq_{\text {left }}$ & $\times$ \\
\hline$s_{2}$ & $\times$ & $=$ & $\geq_{\text {left }}$ & $\times$ \\
\hline$s_{3}$ & $\leq$ left & $\leq_{\text {left }}$ & $=$ & $\times$ \\
\hline$s_{4}$ & $\times$ & $\times$ & $\times$ & $=$ \\
\hline
\end{tabular}

So $s_{1} \geq_{h, l} s_{2} \geq_{h, l} s_{3} \geq_{h, l} s_{4}$. 


\subsection{Infinitesimal coproduct of $\mathcal{H}$}

We define:

$$
\varepsilon:\left\{\begin{aligned}
\mathcal{H} & \longrightarrow K \\
F \in \mathbf{F} & \longrightarrow \delta_{F, 1}
\end{aligned}\right.
$$

Then $\varepsilon$ is clearly an algebra morphism. Moreover, $\varepsilon \circ B^{+}=0$. We also consider:

$$
v:\left\{\begin{aligned}
& K \longrightarrow \mathcal{H} \\
& \lambda \longrightarrow \lambda 1 .
\end{aligned}\right.
$$

Note that $\varepsilon \circ v=I d_{K}$.

Theorem 9 Let $\Delta: \mathcal{H} \longrightarrow\left(\mathcal{H} \otimes \mathcal{H},{ }_{\varepsilon}\right)$ be the unique algebra morphism such that:

$$
\Delta \circ B^{+}=\left(I d \otimes B^{+}+B^{+} \otimes(\nu \circ \varepsilon)\right) \circ \Delta .
$$

Then $(\mathcal{H}, \Delta)$ is an infinitesimal bialgebra. It is graded by the weight.

\section{Proof.}

First step. Let us show that $\varepsilon$ is a counit for $\Delta$. Let $\varphi=(I d \otimes \varepsilon) \circ \Delta$. By composition, $\varphi$ is an algebra endomorphism of $\mathcal{H}$. Moreover:

$$
\begin{aligned}
\varphi \circ B^{+} & =(I d \otimes \varepsilon) \circ\left(I d \otimes B^{+}+B^{+} \otimes(v \circ \varepsilon)\right) \circ \Delta \\
& =\left(I d \otimes\left(\varepsilon \circ B^{+}\right)+B^{+} \otimes(\varepsilon \circ v \circ \varepsilon)\right) \circ \Delta \\
& =B^{+} \circ(I d \otimes \varepsilon) \circ \Delta \\
& =B^{+} \circ \varphi .
\end{aligned}
$$

By unicity in the universal property, $\varphi=I d_{\mathcal{H}}$. So $\varepsilon$ is a right counity for $\Delta$. Let $\phi=(\varepsilon \otimes I d) \circ \Delta$. Then:

$$
\begin{aligned}
\phi \circ B^{+} & =(\varepsilon \otimes I d) \circ\left(I d \otimes B^{+}+B^{+} \otimes(\nu \circ \varepsilon)\right) \circ \Delta \\
& =\left(\varepsilon \otimes B^{+}+\left(\varepsilon \circ B^{+}\right) \otimes(\nu \circ \varepsilon)\right) \circ \Delta \\
& =B^{+} \circ(\varepsilon \otimes I d) \circ \Delta+0 \\
& =B^{+} \circ \phi .
\end{aligned}
$$

By unicity in the universal property, $\phi=I d_{\mathcal{H}}$. So $\varepsilon$ is a counit for $\Delta$. As a consequence, $(\varepsilon \otimes \varepsilon) \circ \Delta=\varepsilon$.

Second step. Let us show that $\Delta$ is coassociative. We consider $\theta=(\Delta \otimes I d) \circ \Delta$. This is an algebra morphism from $\mathcal{H}$ to $\mathcal{H} \otimes \mathcal{H} \otimes \mathcal{H}$. Moreover:

$$
\begin{aligned}
\theta \circ B^{+} & =\left(\Delta \otimes B^{+}+\left(\Delta \circ B^{+}\right) \otimes(\nu \circ \varepsilon)\right) \circ \Delta \\
& =\left(I d \otimes I d \otimes B^{+}+I d \otimes B^{+} \otimes(\nu \circ \varepsilon)+B^{+} \otimes(\nu \circ \varepsilon) \otimes(\nu \circ \varepsilon)\right) \circ \theta .
\end{aligned}
$$


Consider now $\theta^{\prime}=(I d \otimes \Delta) \circ \Delta$. This is also an algebra morphism from $\mathcal{H}$ to $\mathcal{H} \otimes$ $\mathcal{H} \otimes \mathcal{H}$. Moreover:

$$
\begin{aligned}
\theta^{\prime} \circ B^{+}= & \left(I d \otimes\left(\Delta \circ B^{+}\right)+B^{+} \otimes(\Delta \circ v \circ \varepsilon)\right) \circ \Delta \\
= & \left(I d \otimes I d \otimes B^{+}+I d \otimes B^{+} \otimes(v \circ \varepsilon)\right) \circ \theta^{\prime}+\left(B^{+} \otimes((v \otimes v) \circ \varepsilon)\right) \circ \Delta \\
= & \left(I d \otimes I d \otimes B^{+}+I d \otimes B^{+} \otimes(v \circ \varepsilon)\right) \circ \theta^{\prime} \\
& +\left(B^{+} \otimes(\nu \circ \varepsilon) \otimes(\nu \circ \varepsilon)\right) \circ \theta^{\prime} \\
= & \left(I d \otimes I d \otimes B^{+}+I d \otimes B^{+} \otimes(v \circ \varepsilon)+B^{+} \otimes(\nu \circ \varepsilon) \otimes(v \circ \varepsilon)\right) \circ \theta^{\prime} .
\end{aligned}
$$

By unicity in the universal property, $\theta=\theta^{\prime}$, so $\Delta$ is coassociative. As $\Delta: \mathcal{H} \longrightarrow$ $\left(\mathcal{H} \otimes \mathcal{H},{ }_{\varepsilon}\right)$ is a morphism of algebras, $(\mathcal{H}, \Delta)$ is an infinitesimal bialgebra.

Last step. It remains to show that $\Delta$ is homogeneous of degree 0 . Easy induction, using the fact that $I d \otimes B^{+}+B^{+} \otimes(\nu \circ \varepsilon)$ is homogeneous of degree 1 . Note that it can also be proved from proposition 10 .

\section{Remarks.}

1. In other terms, the coproduct $\Delta$ is uniquely defined by the following relations: for all $x, y \in \mathcal{H}$,

$$
\left\{\begin{aligned}
\Delta(1) & =1 \otimes 1, \\
\Delta(x y) & =(x \otimes 1) \Delta(y)+\Delta(x)(1 \otimes y)-x \otimes y, \\
\Delta\left(B^{+}(x)\right) & =\left(I d \otimes B^{+}\right) \circ \Delta(x)+B^{+}(x) \otimes 1 .
\end{aligned}\right.
$$

2. Equivalently, the non unitary coproduct $\tilde{\Delta}$ satisfies the following property: for all $x$ in the augmentation ideal of $\mathcal{H}, \tilde{\Delta} \circ B^{+}(x)=B^{+}(x) \otimes \cdot+\left(I d \otimes B^{+}\right) \circ \tilde{\Delta}(x)$.

\section{Examples.}

$$
\begin{aligned}
& \Delta(\bullet)=\bullet \otimes 1+1 \otimes \cdot, \\
& \Delta(\bullet)=\ldots \otimes 1+1 \otimes \ldots+\cdot \otimes \bullet, \\
& \Delta(\mathfrak{l})=: \otimes 1+1 \otimes !+\bullet \otimes \bullet, \\
& \Delta(\bullet .)=: . \otimes 1+1 \otimes: .+. \otimes \ldots+: \otimes ., \\
& \Delta(V)=V \otimes 1+1 \otimes V+\ldots \otimes \cdot+\cdot \otimes l, \\
& \Delta(\vdots)=\lfloor\otimes 1+1 \otimes \vdots+! \otimes \cdot+\cdot \otimes ! .
\end{aligned}
$$

We now give a combinatorial description of this coproduct. Let $F \in \mathbf{F}$ and $c \in$ $\operatorname{Adm}(F)$. Let $s_{1} \geq_{h, l} \ldots \geq_{h, l} s_{n}$ be the vertices of $F$. We shall say that $c$ is totally admissible if there exists $k \in\{1, \ldots, n\}$ such that the vertices of $P^{c}(F)$ are $s_{1}, \ldots, s_{k}$ and the vertices of $R^{c}(F)$ are $s_{k+1}, \ldots, s_{n}$. The set of totally admissible cuts of $F$ will be denoted $\operatorname{Adm}^{\text {tot }}(F)$.

Proposition 10 Let $F \in \mathbf{F}$. Then $\Delta(F)=\sum_{c \in \operatorname{Adm}^{\text {tot }}(F)} P^{c}(F) \otimes R^{c}(F)+F \otimes 1+1 \otimes F$. 
Proof. Consider $\Delta^{\prime}: \mathcal{H} \longrightarrow \mathcal{H} \otimes \mathcal{H}$, defined by the formula of the proposition 10. It is easy to show that, if $F, G \in \mathbf{F}$ :

$$
\left\{\begin{aligned}
\Delta^{\prime}(1) & =1 \otimes 1, \\
\Delta^{\prime}(F G) & =(F \otimes 1) \Delta^{\prime}(G)+\Delta^{\prime}(F)(1 \otimes G)-F \otimes G, \\
\Delta^{\prime}\left(B^{+}(F)\right) & =B^{+}(F) \otimes 1+\left(I d \otimes B^{+}\right) \circ \Delta^{\prime}(F) .
\end{aligned}\right.
$$

By unicity in theorem $9, \Delta=\Delta^{\prime}$

Let us give a description of the branchs of the totally admissible cuts.

Definition 11 Let $F \in \mathbf{F}$. Let $I$ be a set of vertices of $F$.

1. We shall say that $I$ is an ideal for $\geq_{h i g h}$ if, for all vertices $s, s^{\prime}$ of $F$ :

$$
s \in I \text { and } s^{\prime} \geq_{\text {high }} s \Longrightarrow s^{\prime} \in I \text {. }
$$

2. We shall say that $I$ is an ideal for $\geq_{l e f t}$ if, for all vertices $s, s^{\prime}$ of $F$,

$$
s \in I \text { and } s^{\prime} \geq_{\text {left }} s \Longrightarrow s^{\prime} \in I .
$$

3. We shall say that $I$ is a biideal if $I$ is an ideal for $\geq_{\text {high }}$ and $\geq_{\text {left }}$.

Proposition 12 Let $F \in \mathbf{F}$. Then $\Delta(F)=\sum_{\text {I bideal of } F} I \otimes(F-I)$.

Proof. Similar to the proof of proposition 10 .

Let us make precise the biideals of a forest $F$.

Lemma 13 Let $F \in \mathbf{F}$ and let $s_{1} \geq_{h, l} \ldots \geq_{h, l} s_{n}$ be its vertices. The biideals of $F$ are the sets $I_{k}=\left\{s_{1}, \ldots, s_{k}\right\}$, for $k \in\{0, \ldots, n\}$.

Proof. Let $I$ be a biideal of $F$. Let $k$ be the greater integer such that $s_{k} \in I$. Then $I \subseteq I_{k}$. Let $j \leq k$. Then $s_{j} \geq_{h, l} s_{k}$, so $s_{j} \geq_{h i g h} s_{k}$ or $s_{j} \geq_{l e f t} s_{k}$. As $I$ is a biideal, in both cases $s_{j} \in I$; hence, $I=I_{k}$.

It remains to show that $I_{k}$ is a biideal. Let $s_{j} \in I_{k}$ (so $j \leq k$ ), and $s_{i}$ be a vertex of $F$ such that $s_{i} \geq_{h i g h} s_{j}$ or $s_{i} \geq_{\text {left }} s_{j}$. Then, $s_{i} \geq_{h, l} s_{j}$ so $i \leq j \leq k$, and $s_{i} \in I_{k}$.

Remark. This implies that for any forest $F \in \mathbf{F}$, of weight $n$, for any $1<k<n$, there exists a unique totally admissible cut $c$ such that the weight of $P^{c}(F)$ is equal to $k$.

\subsection{Antipode of $\mathcal{H}$}

As $\mathcal{H}$ is graded, with $\mathcal{H}_{0}=K$, it automatically has an antipode $S$, inductively defined by:

$$
\left\{\begin{array}{l}
S(1)=1 \\
S(x)=-x-S\left(x^{\prime}\right) x^{\prime \prime} \text { if } \varepsilon(x)=0
\end{array}\right.
$$


Because $\mathcal{H}$ is an infinitesimal Hopf algebra, $S$ satisfies $S\left(\operatorname{Ker}(\varepsilon)^{2}\right)=0$, so $S(F)=0$ for all forest $F$ with at least two trees. It remains to give a formula for the antipode of a single tree.

Definition 14 Let $t \in \mathbf{T}$. Let $s$ be the greatest vertex of $t$ for the total order relation $\geq_{h, l}$. In other terms, $s$ is the leave of $t$ which is at most on the left.

1. Let $e$ be an edge of $t$. It will be called a left edge if it is on the path from the root to $s$.

2. Let $c$ be a (possibly empty) cut of $t$. We shall say that $c$ is a left cut if it cuts only left edges.

Let $t \in \mathbf{T}$ and $c$ be a left cut of $t$. The cut $c$ makes $t$ into several trees $t_{1}, \ldots, t_{n}$. These trees are indexed such that, by denoting $r_{i}$ the root of $t_{i}$ for all $i, r_{1} \geq_{h, l}$ $\ldots \geq_{h, l} r_{n}$ in $t$. The forest $t_{1} \ldots t_{n}$ will be denoted $W^{c}(t)$. Moreover, we denote by $n_{c}$ the number of edges which are cut by $c$.

Proposition 15 Let $t \in \mathbf{T}$. Then $S(t)=-\sum_{\text {cleft cut of } t}(-1)^{n_{c}} W^{c}(t)$.

Proof. We prove the result by induction on the weight $n$ of $t$. If $n=1$, then $t=$. and the result is obvious. Suppose the result true for all trees of weight $<n$. We put $t=B^{+}\left(t_{1} \ldots t_{k}\right)$. Two cases are possible.

1. If $k=1$, then:

$$
\begin{aligned}
\Delta(t) & =t \otimes 1+\left(I d \otimes B^{+}\right) \circ \Delta\left(t_{1}\right) \\
& =t \otimes 1+1 \otimes t+t_{1} \otimes \cdot+t_{1}^{\prime} \otimes B^{+}\left(t_{1}^{\prime \prime}\right), \\
S(t) & =-\underbrace{t}_{\begin{array}{c}
(-1)^{n_{c}} W^{c}(t), \\
c \text { empty }
\end{array}}-\underbrace{S\left(t_{1}\right) \cdot}_{\begin{array}{c}
(-1)^{n_{c}} W^{c}(t), \\
c \text { cuts the left edge } \\
\text { from the root }
\end{array}}-\underbrace{S\left(t_{1}^{\prime}\right) B^{+}\left(t_{1}^{\prime \prime}\right) .}_{\begin{array}{c}
(-1)^{n} c W^{c}(t), \\
\begin{array}{c}
(\text { does not cut the left } \\
\text { edge from the root }
\end{array}
\end{array}}
\end{aligned}
$$

2. If $k \geq 2$, then:

$$
\begin{aligned}
\Delta(t)= & t \otimes 1+\left(I d \otimes B^{+}\right) \circ \Delta\left(t_{1} \ldots t_{k}\right) \\
= & t \otimes 1+1 \otimes t+t_{1} \ldots t_{k} \otimes .+\left(t_{1} \ldots t_{k}\right)^{\prime} \otimes B^{+}\left(\left(t_{1} \ldots t_{k}\right)^{\prime \prime}\right) \\
= & t \otimes 1+1 \otimes t+t_{1} \ldots t_{k} \otimes . \\
& +\sum_{i=1}^{k-1} t_{1} \ldots t_{i} \otimes B^{+}\left(t_{i+1} \ldots t_{k}\right)+\sum_{i=1}^{k} t_{1} \ldots t_{i}^{\prime} \otimes B^{+}\left(t_{i}^{\prime \prime} \ldots t_{k}\right) \\
S(t)= & -t-S\left(t_{1} \ldots t_{k}\right) \cdot-\sum_{i=1}^{k-1} S\left(t_{1} \ldots t_{i}\right) B^{+}\left(t_{i+1} \ldots t_{k}\right) \\
& \quad-\sum_{i=1}^{k} S\left(t_{1} \ldots t_{i}^{\prime}\right) B^{+}\left(t_{i}^{\prime \prime} \ldots t_{k}\right)
\end{aligned}
$$




$$
\begin{aligned}
& =-t-S\left(t_{1}\right) B^{+}\left(t_{2} \ldots t_{k}\right)-S\left(t_{1}^{\prime}\right) B^{+}\left(t_{1}^{\prime \prime} \ldots t_{k}\right) \\
& =-\underbrace{t}_{\begin{array}{c}
(-1)^{n_{c} W^{c}}(t), \\
c \text { empty }
\end{array}}-\underbrace{S\left(t_{1}\right) B^{+}\left(t_{2} \ldots t_{k}\right)}_{\begin{array}{c}
(-1)^{n_{c}} W^{c}(t), \\
c \text { cuts the left tedge } \\
\text { from the root }
\end{array}}-\underbrace{S\left(t_{1}^{\prime}\right) B^{+}\left(t_{1}^{\prime \prime} t_{2} \ldots t_{k}\right)}_{\begin{array}{c}
(-1)^{n_{c}} W^{c}(t), \\
c \text { does not cut the left edge } \\
\text { from the root }
\end{array}} .
\end{aligned}
$$

So the result holds for all forests.

\section{Examples.}

$$
\begin{aligned}
& S(\bullet)=-\bullet, \\
& S(\bullet)=-\mathfrak{l}+\ldots, \\
& S(V)=-V+\ldots \text {, } \\
& S(\vdots)=-\vdots+. !+! . \ldots, \\
& S(\ddot{V})=-\ddot{V}+\cdot \mathcal{V} \text {, }
\end{aligned}
$$

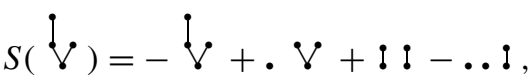

$$
\begin{aligned}
& S(\dot{v})=-\dot{v}+. \dot{l} \text {, } \\
& S(\vdots)=-\vdots+\ldots+! \vdots+\lfloor-\ldots !-. ! .-. !+\ldots . .
\end{aligned}
$$

\subsection{Infinitesimal universal property}

Theorem 16 (Infinitesimal universal property) Let $A$ be an infinitesimal Hopf algebra and let $L: A \longrightarrow A$ satisfying $\Delta(L(x))=L(x) \otimes 1+(I d \otimes L) \circ \Delta(x)$. Then there exists a unique infinitesimal Hopf algebra morphism $\phi: \mathcal{H} \longrightarrow A$ such that $\phi \circ B^{+}=L \circ \phi$.

Proof. By the universal property of $\mathcal{H}$, there exists a unique algebra morphism $\phi$ satisfying $\phi \circ B^{+}=L \circ \phi$. Let us show that it is also a coalgebra morphism.

First step. We first show that $\varepsilon \circ L=0$. Let $a \in A$.

$$
\begin{aligned}
\varepsilon \circ L(a) & =(\varepsilon \otimes \varepsilon) \circ \Delta \circ L(a) \\
& =(\varepsilon \otimes \varepsilon)\left(L(a) \otimes 1+a^{(1)} \otimes L\left(a^{(2)}\right)\right) \\
& =\varepsilon \circ L(a)+\varepsilon\left(a^{(1)}\right) \varepsilon \circ L\left(a^{(2)}\right) \\
& =\varepsilon \circ L(a)+\varepsilon \circ L(a) .
\end{aligned}
$$

So $\varepsilon \circ L(a)=0$.

Second step. We consider $X=\{x \in \mathcal{H} / \varepsilon \circ \phi(a)=\varepsilon(x)\}$. As $\varepsilon \circ \phi$ and $\varepsilon$ are both algebra morphisms, $X$ is a subalgebra of $\mathcal{H}$. Let $x \in \mathcal{H}$. Then:

$$
(\varepsilon \circ \phi)\left(B^{+}(x)\right)=\varepsilon \circ L \circ \phi(x)=0=\varepsilon\left(B^{+}(x)\right) .
$$


So $\operatorname{Im}\left(B^{+}\right) \subseteq X$. As $X$ is a subalgebra, $X=\mathcal{H}$, and $\varepsilon \circ \phi=\varepsilon$.

Third step. We consider $Y=\{x \in \mathcal{H} / \Delta \circ \phi(x)=(\phi \otimes \phi) \circ \Delta\}$. As $\Delta \circ \phi$ and $(\phi \otimes \phi) \circ \Delta$ are algebra morphisms from $\mathcal{H}$ to $(A \otimes A, . \varepsilon), Y$ is a subalgebra of $\mathcal{H}$. Let $x \in Y$.

$$
\begin{aligned}
(\Delta \circ \phi)\left(B^{+}(x)\right) & =\Delta \circ L \circ \phi(x) \\
& =L \circ \phi(x) \otimes 1+\phi(x)^{(1)} \otimes L\left(\phi(x)^{(2)}\right) \\
& =\phi \circ B^{+}(x) \otimes 1+\phi\left(x^{(1)}\right) \otimes L\left(\phi\left(x^{(2)}\right)\right) \\
& =\phi \circ B^{+}(x) \otimes \phi(1)+\phi\left(x^{(1)}\right) \otimes \phi\left(B^{+}\left(x^{(2)}\right)\right) \\
& =(\phi \otimes \phi)\left(B^{+}(x) \otimes 1+x^{(1)} \otimes B^{+}\left(x^{(2)}\right)\right) \\
& =(\phi \otimes \phi) \circ \Delta\left(B^{+}(x)\right) .
\end{aligned}
$$

So $B^{+}(x) \in Y$. As $Y$ is a subalgebra of $\mathcal{H}$ stable under $B^{+}, Y=\mathcal{H}$. Hence, $\phi$ is a coalgebra morphism.

\subsection{A pairing on $\mathcal{H}$}

Definition 17 The linear map $\gamma$ is defined by:

$$
\gamma:\left\{\begin{array}{c}
\mathcal{H} \longrightarrow \mathcal{H} \\
t_{1} \ldots t_{n} \in \mathbf{F} \longrightarrow \delta_{t_{1}, \bullet} t_{2} \ldots t_{n} .
\end{array}\right.
$$

\section{Lemma 18}

1. $\gamma$ is homogeneous of degree -1 .

2. For all $x, y \in \mathcal{H}, \gamma(x y)=\gamma(x) y+\varepsilon(x) \gamma(y)$.

3. $\operatorname{Ker}(\gamma) \cap \operatorname{Prim}(\mathcal{H})=(0)$.

\section{Proof.}

1. Trivial.

2. Immediate for $x, y \in \mathbf{F}$, separating the cases $x=1$ and $x \neq 1$.

3. Let us take $p \in \operatorname{Ker}(\gamma)$, non-zero, and primitive. Then $p$ can be written as:

$$
p=\sum_{F \in \mathbf{F}} a_{F} F
$$

Let us choose a forest $F=t_{1} \ldots t_{n}$ such that:

(a) $a_{F} \neq 0$.

(b) If $G=t_{1}^{\prime} \ldots t_{m}^{\prime} \in \mathbf{F}$ is such that $a_{G} \neq 0$, then $m \leq n$. If moreover $m=n$, we put $t_{1}=B^{+}\left(s_{1} \ldots s_{k}\right)$ and $t_{1}^{\prime}=B^{+}\left(s_{1}^{\prime} \ldots s_{l}^{\prime}\right)$; then $k \leq l$.

Suppose that $t_{1} \neq \ldots$. Then $k \neq 0$. We consider the cut $c$ on the edge from the root of $t_{1}$ to the root of $s_{1}$. This is a totally admissible cut, so $s_{1} \otimes B^{+}\left(s_{2} \ldots s_{k}\right) t_{2} \ldots t_{n}$ appears in $\Delta(F)$. Because $p$ is primitive, there exists another forest $G$ such that $a_{G} \neq 0$ and $s_{1} \otimes B^{+}\left(s_{2} \ldots s_{k}\right) t_{2} \ldots t_{n}$ appears in $\Delta(G)$. Three cases are possible: 
(a) $G=s_{1} B^{+}\left(s_{2} \ldots s_{k}\right) t_{2} \ldots t_{n}$ : contradicts the maximality of $n$ for $F$.

(b) $G=F$ : contradicts that $G \neq F$.

(c) $G$ is obtained by grafting $s_{1}$ on a vertex of $s_{2}$ : contradicts the minimality of $k$.

In every case, we obtain a contradiction. So $t_{1}=$. Hence, $\gamma(p) \neq 0$.

Theorem 19 There exists a unique pairing $\langle-,-\rangle: \mathcal{H} \times \mathcal{H} \longrightarrow K$, satisfying:

1. $\langle 1, x\rangle=\varepsilon(x)$ for all $x \in \mathcal{H}$.

2. $\langle x y, z\rangle=\langle y \otimes x, \Delta(z)\rangle$ for all $x, y, z \in \mathcal{H}$.

3. $\left\langle B^{+}(x), y\right\rangle=\langle x, \gamma(y)\rangle$ for all $x, y \in \mathcal{H}$.

Moreover:

4. $\langle-,-\rangle$ is symmetric and non-degenerate.

5. If $x$ and $y$ are homogeneous of different weights, $\langle x, y\rangle=0$.

6. $\langle S(x), y\rangle=\langle x, S(y)\rangle$ for all $x, y \in \mathcal{H}$.

\section{Proof.}

Unicity. Assertions 1-3 entirely determine $\langle F, G\rangle$ for $F, G \in \mathbf{F}$ by induction on the weight.

Existence. We consider the graded infinitesimal Hopf algebra $A=\mathcal{H}^{*, o p, c o p}$. As $\gamma$ is homogeneous of degree -1 , it can be transposed in a linear map: $L=\gamma^{*}: A \longrightarrow$ $A$. This linear map is homogeneous of degree 1 . Let $f \in A$, and $x, y \in \mathcal{H}$.

$$
\begin{aligned}
((\Delta \circ L)(f))(x \otimes y) & =L(f)(y x) \\
& =f(\gamma(y x)) \\
& =f(\gamma(y) x+\varepsilon(y) \gamma(x)) \\
& =\Delta(f)(x \otimes \gamma(y))+(f \otimes 1)(\gamma(x) \otimes y) \\
& =((I d \otimes L) \circ \Delta(f)+L(f) \otimes 1)(x \otimes y) .
\end{aligned}
$$

So $\Delta \circ L(f)=(I d \otimes L) \circ \Delta(f)+L(f) \otimes 1$ for all $f \in A$. By the infinitesimal universal property, there exists a unique infinitesimal Hopf algebra morphism $\phi$ : $\mathcal{H} \longrightarrow A$, such that $\phi \circ B^{+}=L \circ \phi$. We then put $\langle x, y\rangle=\phi(x)(y)$ for all $x, y \in \mathcal{H}$. Let us show that this pairing satisfies 1-6.

1. For all $x \in \mathcal{H},\langle 1, x\rangle=\phi(1)(x)=\varepsilon(x)$.

2. For all $x, y, z \in \mathcal{H}$ :

$$
\langle x y, z\rangle=\phi(x y)(z)=(\phi(x) \phi(y))(z)=(\phi(y) \otimes \phi(x))(\Delta(z))=\langle y \otimes x, \Delta(z)\rangle .
$$

3. For all $x, y \in \mathcal{H}$ :

$$
\left\langle B^{+}(x), y\right\rangle=\left(\phi \circ B^{+}(x)\right)(y)=(L \circ \phi(x))(y)=\phi(x)(\gamma(y))=\langle x, \gamma(y)\rangle .
$$

5. As $L$ is homogeneous of degree $1, \phi$ is homogeneous of degree 0 . This implies 5 . 
6. As $\phi$ is an infinitesimal Hopf algebra morphism and $S^{*}$ is the antipode of $A$, $S^{*} \circ \phi=\phi \circ S$. Hence, for all $x, y \in \mathcal{H}$ :

$$
\langle S(x), y\rangle=\phi(S(x))(y)=\left(S^{*} \circ \phi(x)\right)(y)=\phi(x)(S(y))=\langle x, S(y)\rangle .
$$

4. Let us first show that $\langle-,-\rangle$ is symmetric. We put $\langle x, y\rangle^{\prime}=\langle y, x\rangle$ for all $x, y \in$ $\mathcal{H}$. Let us show that $\langle-,-\rangle^{\prime}$ satisfies $1-3$. For all $x \in \mathcal{H}$, as $f(1)=\varepsilon(f)$ for all $f \in \mathcal{H}^{*}$ :

$$
\langle 1, x\rangle^{\prime}=\langle x, 1\rangle=\phi(x)(1)=\varepsilon(\phi(x))=\varepsilon(x) .
$$

For all $x, y, z \in \mathcal{H}$, as $\phi$ is a coalgebra morphism:

$$
\begin{aligned}
\langle x y, z\rangle^{\prime} & =\langle z, x y\rangle \\
& =\phi(z)(x y) \\
& =(\Delta \circ \phi(z))(y \otimes x) \\
& =((\phi \otimes \phi) \circ \Delta(z))(y \otimes x) \\
& =\langle\Delta(z), y \otimes x\rangle \\
& =\langle y \otimes x, \Delta(z)\rangle^{\prime} .
\end{aligned}
$$

Let us show 3 for $\langle-,-\rangle^{\prime}$ with $y \in \mathbf{F}$, by induction on $n=$ weight $(y)$. If $n=0$, then $y=1$ :

$$
\left\langle B^{+}(x), y\right\rangle^{\prime}=\left\langle 1, B^{+}(x)\right\rangle=\varepsilon \circ B^{+}(x)=0=\langle x, \gamma(y)\rangle^{\prime} .
$$

Suppose the result true for every forest $F$ of weight $<n$. Two cases are possible: - $y=B^{+}(z)$. We can restrict to the case where $x$ is also a forest. Then:

$$
\left\langle B^{+}(x), y\right\rangle^{\prime}=\left\langle B^{+}(z), B^{+}(x)\right\rangle=\left\langle z, \gamma \circ B^{+}(x)\right\rangle=\delta_{x, 1}\langle z, 1\rangle=\varepsilon(x) \varepsilon(z) .
$$

Moreover, $\langle x, \gamma(y)\rangle^{\prime}=\left\langle\gamma \circ B^{+}(z), x\right\rangle=\delta_{z, 1}\langle 1, x\rangle=\varepsilon(z) \varepsilon(x)$.

- $y$ is a forest with at least two trees. Then $y$ can be written $y=y_{1} y_{2}$, with the induction hypothesis avalaible for $y_{1}$ and $y_{2}$. Then:

$$
\begin{aligned}
\left\langle B^{+}(x), y\right\rangle^{\prime} & =\left\langle y_{1} y_{2}, B^{+}(x)\right\rangle \\
& =\left\langle y_{2} \otimes y_{1}, \Delta \circ B^{+}(x)\right\rangle \\
& =\left\langle y_{2} \otimes y_{1}, B^{+}(x) \otimes 1+\left(I d \otimes B^{+}\right) \circ \Delta(x)\right\rangle \\
& =\left\langle\gamma\left(y_{2}\right) \varepsilon\left(y_{1}\right), x\right\rangle+\left\langle y_{2} \otimes \gamma\left(y_{1}\right), \Delta(x)\right\rangle \\
& =\left\langle\varepsilon\left(y_{1}\right) \gamma\left(y_{2}\right)+\gamma\left(y_{1}\right) y_{2}, x\right\rangle \\
& =\langle\gamma(y), x\rangle \\
& =\langle x, \gamma(y)\rangle^{\prime} .
\end{aligned}
$$

So $\langle-,-\rangle^{\prime}$ satisfies $1-3$. By unicity, $\langle-,-\rangle^{\prime}=\langle-,-\rangle$, so $\langle-,-\rangle$ is symmetric. 
Note that this implies that for all $x, y \in \mathcal{H}$ :

$$
\begin{aligned}
(\phi \circ \gamma(x))(y) & =\langle\gamma(x), y\rangle=\langle y, \gamma(x)\rangle=\left\langle B^{+}(y), x\right\rangle=\left\langle x, B^{+}(y)\right\rangle \\
& =(\phi(x))\left(B^{+}(y)\right) .
\end{aligned}
$$

So $\phi \circ \gamma=\left(B^{+}\right)^{*} \circ \phi$.

It remains to prove that $\langle-,-\rangle$ is non degenerate. It is equivalent to show that $\phi$ is one-to-one. Suppose that it is not. Let us choose a non-zero element $p \in \operatorname{Ker}(\phi)$ of lowest degree. As $\phi$ is a coalgebra morphism, its kernel is a coideal, so $p \in$ $\operatorname{Prim}(\mathcal{H})$. By lemma 18-3, $\gamma(p) \neq 0$. Moreover, $\phi \circ \gamma(p)=\left(B^{+}\right)^{*} \circ \phi(p)=0$. So $\gamma(p) \in \operatorname{Ker}(\phi)$, is non-zero, of degree strictly smaller than $p$ : this contradicts the choice of $p$. So $\phi$ is one-to-one.

Remark. Similarly with the usual case, it is possible to define a pairing between $\mathcal{H}$ and itself, using the linear map:

$$
\gamma^{\prime}:\left\{\begin{array}{c}
\mathcal{H} \longrightarrow \mathcal{H} \\
t_{1} \ldots t_{n} \in \mathbf{F} \longrightarrow \delta_{t_{n}, \bullet}, t_{1} \ldots t_{n-1} .
\end{array}\right.
$$

Unhappily, this pairing is degenerate: it is for example not difficult to show that the primitive element $\vee-\ldots$ : belongs to $\mathcal{H}^{\perp}$.

Definition 20 We denote by $\left(f_{F}\right)_{F \in \mathbf{F}}$ the dual basis of the basis of forests. In other terms, for all $F \in \mathbf{F}, f_{F}$ is defined by $\left\langle f_{F}, G\right\rangle=\delta_{F, G}$, for all forest $G \in \mathbf{F}$.

\section{Proposition 21}

1. For all forest $F \in \mathbf{F}, B^{+}\left(f_{F}\right)=f . F$.

2. For all forest $F \in \mathbf{F}$ :

$$
\gamma\left(f_{F}\right)=\left\{\begin{array}{r}
0 \text { if } F \notin \mathbf{T}, \\
f_{B^{-}(F)} \text { if } F \in \mathbf{T},
\end{array}\right.
$$

where $B^{-}(F)$ is the forest obtained by deleting the root of $F$.

3. For all forest $F \in \mathbf{F}, \Delta\left(f_{F}\right)=\sum_{\substack{F_{1}, F_{2} \in \mathbf{F} \\ F_{1} F_{2}=F}} f_{F_{2}} \otimes f_{F_{1}}$.

\section{Proof.}

1. Let $G \in \mathbf{F}$. Then:

$$
\begin{aligned}
\left\langle B^{+}\left(f_{F}\right), G\right\rangle & =\left\langle f_{F}, \gamma(G)\right\rangle \\
& =\left\{\begin{array}{l}
0 \text { if } G \text { is not of the form } \bullet H, \\
\delta_{F, H} \text { if } G=\cdot H,
\end{array}\right. \\
& =\delta_{\bullet}, G \\
& =\left\langle f_{\bullet}, G\right\rangle .
\end{aligned}
$$

As $\langle-,-\rangle$ is non-degenerate, $B^{+}\left(f_{F}\right)=f_{\cdot F}$. 
2. Suppose first that $F$ is not a tree. Then, for all $G \in \mathbf{F}$ :

$$
\left\langle\gamma\left(f_{F}\right), G\right\rangle=\left\langle f_{F}, B^{+}(G)\right\rangle=\delta_{F, B^{+}(G)}=0 .
$$

So $\gamma\left(f_{F}\right)=0$. Suppose now that $F$ is a tree. Then, for all $G \in \mathbf{F}$ :

$$
\left\langle\gamma\left(f_{F}\right), G\right\rangle=\delta_{F, B^{+}(G)}=\delta_{B^{-}(F), G}=\left\langle f_{B^{-}(F)}, G\right\rangle .
$$

So $\gamma\left(f_{F}\right)=f_{B^{-}(F)}$.

3. Indeed, for all forests $G_{1}, G_{2} \in \mathbf{F}$,

$$
\left\langle\Delta\left(f_{F}\right), G_{1} \otimes G_{2}\right\rangle=\left\langle f_{F}, G_{2} G_{1}\right\rangle=\delta_{F, G_{2} G_{1}}=\sum_{\substack{F_{1}, F_{2} \in \mathbf{F} \\ F_{1} F_{2}=F}}\left\langle f_{F_{2}} \otimes f_{F_{1}}, G_{1} \otimes G_{2}\right\rangle .
$$

As $\langle-,-\rangle$ is non-degenerate, this proves the last point.

Proposition 22 The familly $\left(f_{t}\right)_{t \in \mathbf{T}}$ is a basis of $\operatorname{Prim}(\mathcal{H})$.

Proof. Immediate corollary of proposition 21-3.

As an example of decomposition in the dual basis, we give the following result:

Corollary 23 For all $n \in \mathbb{N}, \cdot^{n}=\sum_{\substack{F \in \mathbf{F} \\ \text { weight }(F)=n}} f_{F}$.

Proof. For all $n \in \mathbb{N}^{*}$, we can put, by homogeneity:

$$
\bullet^{n}=\sum_{\substack{F \in \mathbf{F} \\ \text { weight }(F)=n}} a_{F} f_{F} .
$$

We define $X=\left\{F \in \mathbf{F} / a_{F}=1\right\}$. Note that $f_{1}=1$, so $1 \in X$. Let $F_{1}, F_{2} \in X$. Then, if $n=$ weight $\left(F_{1} F_{2}\right)$ :

$$
\begin{aligned}
a_{F_{1} F_{2}} & =\left\langle\bullet^{n}, F_{1} F_{2}\right\rangle \\
& =\left\langle\Delta\left(\bullet^{n}\right), F_{2} \otimes F_{1}\right\rangle \\
& =\left\langle\sum_{i=0}^{n} \cdot^{i} \otimes \cdot^{n-i}, F_{2} \otimes F_{1}\right\rangle \\
& =\left\langle\cdot \bullet^{\text {eight }\left(F_{2}\right)} \otimes \cdot \text { weight }\left(F_{1}\right), F_{2} \otimes F_{1}\right\rangle+0 \\
& =a_{F_{2}} a_{F_{1}} \\
& =1 .
\end{aligned}
$$

So $F_{1} F_{2} \in X$. Moreover, if $m=$ weight $\left(F_{1}\right)$ :

$$
a_{B^{+}\left(F_{1}\right)}=\left\langle\bullet^{m+1}, B^{+}\left(F_{1}\right)\right\rangle=\left\langle\gamma\left(\bullet^{m+1}\right), F_{1}\right\rangle=\left\langle\bullet^{m}, F_{1}\right\rangle=a_{F_{1}}=1 .
$$

So $B^{+}\left(F_{1}\right) \in X$. Hence, $1 \in X$, and $X$ is stable by product and by $B^{+}$. So $X=\mathbf{F}$. 


\subsection{Combinatorial interpretation of the pairing}

Notation. Let $F \in \mathbf{F}$. We denote by $V \operatorname{ert}(F)$ the set of vertices of $F$.

Theorem 24 Let $F, G \in \mathbf{F}$. Let $S(F, G)$ be the set of bijections $\sigma: \operatorname{Vert}(F) \longrightarrow$ $\operatorname{Vert}(G)$ such that, for all vertices $x, y$ of $F$ :

1. $(x \leq$ high $y) \Longrightarrow\left(\sigma(x) \geq_{\text {left }} \sigma(y)\right)$.

2. $\left(x \leq_{\text {left }} y\right) \Longrightarrow\left(\sigma(x) \geq_{h, l} \sigma(y)\right)$.

3. $\left(\sigma(x) \leq_{\text {high }} \sigma(y)\right) \Longrightarrow\left(x \geq_{\text {left }} y\right)$.

4. $\left(\sigma(x) \leq_{\text {left }} \sigma(y)\right) \Longrightarrow\left(x \geq_{h, l} y\right)$.

Then $\langle F, G\rangle=\operatorname{card}(S(F, G))$.

Proof. If $F$ and $G$ have different weights, as $\langle-,-\rangle$ is homogeneous, then $\langle F, G\rangle=0$ and $S(F, G)$ is empty, so the result holds. Let us suppose now that $F$ and $G$ have the same weight $n$ and let us proceed by induction on $n$. If $n=0$, then $F=G=1$, and the result holds. For the hereditary, we have the following cases.

1. $F=B^{+}\left(F_{1}\right)$. We have the two following subcases.

(a) $G$ is not of the form $\cdot G_{1}$. Then $\gamma(G)=0$, so $\langle F, G\rangle=\left\langle B^{+}\left(F_{1}\right), G\right\rangle=$ $\left\langle F_{1}, \gamma(G)\right\rangle=0$. Let us assume that $S(F, G)$ is not empty, and let $\sigma \in S(F, G)$. Let $r$ be the root of $F$. For all $x \in \operatorname{vert}(F), x \geq_{\text {high }} r$, so $\sigma(r) \geq_{\text {left }} \sigma(x)$. Hence, as $\sigma$ is epic, $G$ is of the form.$G_{1}$ : contradiction. So $S(F, G)=\emptyset$, and the result holds.

(b) $G={ } G_{1}$. Then $\langle F, G\rangle=\left\langle F_{1}, G_{1}\right\rangle$. Let $\sigma \in S(F, G)$. As in the preceding point, $\sigma(r)$ is the vertex of ., so we can consider the linear map:

$$
\Psi:\left\{\begin{aligned}
S(F, G) & \longrightarrow S\left(F_{1}, G_{1}\right) \\
\sigma & \longrightarrow \sigma_{\mid \operatorname{Vert}}\left(F_{1}\right)
\end{aligned}\right.
$$

It is obviously injective. Let us show it is surjective. Let $\sigma_{1} \in S\left(F_{1}, G_{1}\right)$, and let $\sigma: \operatorname{Vert}(F) \longrightarrow V \operatorname{ert}(G)$ extending $\sigma_{1}$ by sending the root of $F$ to the vertex of . . Let us show that $\sigma \in S(F, G)$. Let $x, y \in \operatorname{Vert}(F)$. We can suppose they are distinct.

- If $x \leq \leq_{\text {high }} y$, two cases are possible. If $x$ is the root of $F$, then $\sigma(x) \geq_{\text {left }}$ $\sigma(y)$. If not, then $x$ and $y$ are vertices of $F_{1}$, so $\sigma(x) \geq_{\text {left }} \sigma(y)$ in $G_{1}$, hence in $G$.

- If $x \leq_{\text {left }} y$, then both of them are vertices of $F_{1}$, so $\sigma(x) \geq_{h, l} \sigma(y)$ in $G_{1}$, hence in $G$.

- If $\sigma(x) \leq_{\text {high }} \sigma(y)$, then $\sigma(x)$ and $\sigma(y)$ are vertices of $G_{1}$, so $x \geq_{\text {left }} y$ in $F_{1}$, hence in $F$.

- If $\sigma(x) \leq_{\text {left }} \sigma(y)$, then two cases are possible. If $\sigma(y)$ is the vertex of ., then $y$ is the root of $F$, so $x \geq_{h, l} y$. If not, then $\sigma(x)$ and $\sigma(y)$ are vertices of $G_{1}$, so $x \geq_{h, l} y$ in $F_{1}$, hence in $F$.

So $\sigma \in S(F, G)$, and $\Psi(\sigma)=\sigma_{1}$. So $\Psi$ is a bijection. As a consequence:

$$
\operatorname{card}(S(F, G))=\operatorname{card}\left(S\left(F_{1}, G_{1}\right)\right)=\left\langle F_{1}, G_{1}\right\rangle=\langle F, G\rangle .
$$


2. $F=F_{1} F_{2}$, with $F_{1}, F_{2} \neq 1$. Let $J$ be the unique biideal of $G$ with the same weight as $F_{2}$. Then:

$$
\langle F, G\rangle=\left\langle F_{2} \otimes F_{1}, \sum_{I \text { biideal of } G} I \otimes(G-I)\right\rangle=\left\langle F_{2}, J\right\rangle\left\langle F_{1}, G-J\right\rangle .
$$

Let $\sigma \in S(F, G)$. Let $\sigma(x) \in \sigma\left(\operatorname{Vert}\left(F_{2}\right)\right)$, and $y^{\prime} \in \operatorname{vert}(G)$, such that $y^{\prime} \geq_{h i g h}$ $\sigma(x)$ or $y^{\prime} \geq_{\text {left }} \sigma(x)$. As $\sigma$ is surjective, we put $y^{\prime}=\sigma(y)$. Then $x \geq_{\text {high }} y$ or $x \geq_{h, l} y$. In both cases, as $x \in \operatorname{Vert}\left(F_{2}\right), y \in \operatorname{Vert}\left(F_{2}\right)$. So $\sigma\left(\operatorname{Vert}\left(F_{2}\right)\right)$ is a biideal of $G$. Considering its weight, it is $J$. We can consider the linear map:

$$
\Phi:\left\{\begin{aligned}
S(F, G) & \longrightarrow S\left(F_{1}, G-J\right) \times S\left(F_{2}, J\right) \\
\sigma & \longrightarrow\left(\sigma_{\mid \operatorname{vert}\left(F_{1}\right)}, \sigma_{\mid \operatorname{vert}\left(F_{2}\right)}\right) .
\end{aligned}\right.
$$

It is clearly injective. Let be $\left(\sigma_{1}, \sigma_{2}\right) \in S\left(F_{1}, G-J\right) \times S\left(F_{2}, J\right)$ and let $\sigma$ : $V \operatorname{ert}(F) \longrightarrow V \operatorname{ert}(G)$, such that $\sigma_{\mid \operatorname{vert}\left(F_{i}\right)}=\sigma_{i}$ for $i=1,2$. Let us show that $\sigma \in S(F, G)$. Let $x, y \in \operatorname{Vert}(F)$.

- If $x \leq_{\text {high }} y$, then $x, y \in F_{1}$ or $x, y \in F_{2}$. So $x \geq_{\text {left }} y$ in $J$ or in $G-J$, hence in $G$.

- If $x \leq_{\text {left }} y$, two cases are possible. If $x, y \in F_{1}$ or $x, y \in F_{2}$, then $x \geq_{h, l} y$ in $J$ or in $G-J$, hence in $G$. If $x \in \operatorname{Vert}\left(F_{2}\right)$ and $y \in \operatorname{Vert}\left(F_{1}\right)$, then $\sigma(x) \in J$ and $\sigma(y) \in G-J$. As $J$ is a biideal, $\sigma(y) \geq_{h, l} \sigma(x)$ is impossible. As $\geq_{h, l}$ is a total order, $\sigma(x) \geq_{h, l} \sigma(y)$.

- If $\sigma(x) \leq_{h i g h} \sigma(y)$, two cases are possible. If $\sigma(x), \sigma(y) \in J$ or $\sigma(x), \sigma(y) \in$ $G-J$, then $x \geq_{\text {left }} y$ in $F_{1}$ or in $F_{2}$, hence in $F$. If $\sigma(x) \in G-J$ and $\sigma(y) \in J$, then $x \in \operatorname{vert}\left(F_{1}\right)$ and $y \in \operatorname{vert}\left(F_{2}\right)$, so $x \geq$ left $y$.

- If $\sigma(x) \leq_{\text {left }} \sigma(y)$, two cases are possible. If $\sigma(x), \sigma(y) \in J$ or $\sigma(x), \sigma(y) \in$ $G-J$, then $x \geq_{h, l} y$ in $F_{1}$ or in $F_{2}$, hence in $F$. If $\sigma(x) \in G-J$ and $\sigma(y) \in J$, then $x \in \operatorname{vert}\left(F_{1}\right)$ and $y \in \operatorname{vert}\left(F_{2}\right)$, so $x \geq_{h, l} y$.

So $\sigma \in S(F, G)$, and $\Phi(\sigma)=\left(\sigma_{1}, \sigma_{2}\right)$. So $\Phi$ is a bijection. Hence:

$$
\begin{aligned}
\langle F, G\rangle & =\left\langle F_{2}, J\right\rangle\left\langle F_{1}, G-J\right\rangle=\operatorname{card}\left(S\left(F_{1}, G-J\right)\right) \operatorname{card}\left(S\left(F_{2}, J\right)\right) \\
& =\operatorname{card}(S(F, G)) .
\end{aligned}
$$

So the result holds for all $n$.

\section{Remarks.}

1. There is obviously a bijection:

$$
\left\{\begin{aligned}
S(F, G) & \longrightarrow S(G, F) \\
\sigma & \longrightarrow \sigma^{-1}
\end{aligned}\right.
$$

This gives another proof of the symmetry of $\langle-,-\rangle$.

2. Let us assume that $S(F, G)$ is not empty, and let $\sigma \in S(F, G)$. By definition, if $x \leq_{h, l} y$ in $\operatorname{Vert}(F)$, then $\sigma(x) \geq_{h, l} \sigma(y)$ in $\operatorname{Vert}(G)$, so $\sigma$ is the unique orderreversing bijection from $\left(V \operatorname{ert}(F), \geq_{h, l}\right)$ to $\left(\operatorname{Vert}(G), \geq_{h, l}\right)$. So, for any forests $F, G \in \mathbf{F},\langle F, G\rangle=0$ or 1 . 


\section{Poset of forests and applications}

3.1 Partial order on $\mathbf{F}$

Notations. Let $n \in \mathbb{N}$. The set of planar forests with $n$ vertices will be denoted by $\mathbf{F}(n)$.

Definition 25 Let $F \in \mathbf{F}$.

1. An admissible transformation on $F$ is a local transformation of one of the following types (the part of $F$ which is not in the frame remains unchanged):

First kind:
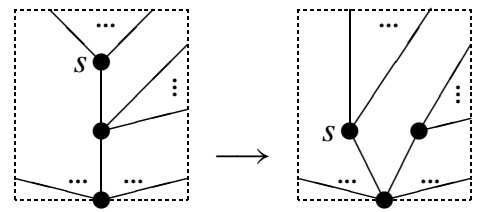

Second kind:
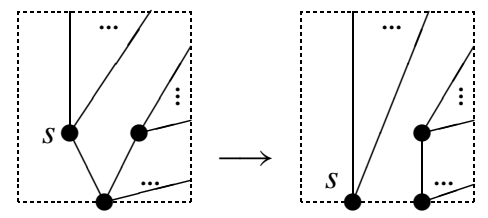

Such a transformation will be said to hold on the vertex $s$.

2. Let $s_{1} \geq_{h, l} \ldots \geq_{h, l} s_{n}$ be the vertices of $F$. An admissible transformation on $F$ will be said to be an $i$-transformation if it is applied at the vertex $s_{i}$.

Example. Let $t=\dot{V}$. The 1-transformation turns $t$ into $\vee$. The 2-transformation turns $t$ into $:$ : There are no 3 - and 4-transformation.

Definition 26 Let $I \subseteq \mathbb{N}^{*}$. Let $F, G \in \mathbf{F}$. We shall say that $F \leq_{I} G$ if there exists a finite sequence $F_{0}, \ldots, F_{k}$ of elements of $\mathbf{F}$ such that:

1. For all $i \in\{0, \ldots, k-1\}, F_{i+1}$ is obtained from $F_{i}$ by a $j$-transformation, for a certain $j \in I$.

2. $F_{0}=F$.

3. $F_{k}=G$.

Proposition 27 For all $I \subseteq \mathbb{N}^{*}, \leq_{I}$ is a partial order $\mathbf{F}$.

Proof. Indeed, $\leq_{I}$ is transitive and reflexive. Let $F, G$ be two forests, such that $F \leq_{I}$ $G$ and $G \leq_{I} F$. We put:

$$
\left\{\begin{array}{l}
n_{F}=\sum_{s \text { vertex of } F} \text { height }(s), \\
n_{G}=\sum_{s \text { vertex of } G} \operatorname{height}(s) .
\end{array}\right.
$$


As $F \leq_{I} G$, there exists $F_{0}, \ldots, F_{k} \in \mathbf{F}$, such that $F_{0}=F, F_{k}=G$, and $F_{i+1}$ is obtained from $F_{i}$ by an admissible transformation. Each admissible transformation decreases the height of at least one vertex by 1 , so $n_{G} \leq n_{F}-k$, so $n_{G} \leq n_{F}$. As $G \leq_{I} F$, in the same way, $n_{F} \leq n_{G}$, so $n_{F}=n_{G}$, and $k=0$. As a consequence, $F=G$.

\section{Remarks.}

1. $F$ and $G$ are comparable for $\leq \emptyset$ if, and only if, they are equal.

2. We shall denote $\leq$ instead of $\leq_{\mathbb{N}}$. This order $\leq$ is the order generated by all the admissible transformations.

3. If $F$ and $G \in \mathbf{F}$ are comparable for one of these orders, they have the same weight. So $\mathbf{F}=\bigcup_{n \in \mathbb{N}} \mathbf{F}(n)$ as a poset.

Examples. The posets $\mathbf{F}(0)$ and $\mathbf{F}(1)$ are reduced to a single element. Here are the Hasse graphs of $(\mathbf{F}(2), \leq),(\mathbf{F}(3), \leq)$ and $(\mathbf{F}(4), \leq)$ :
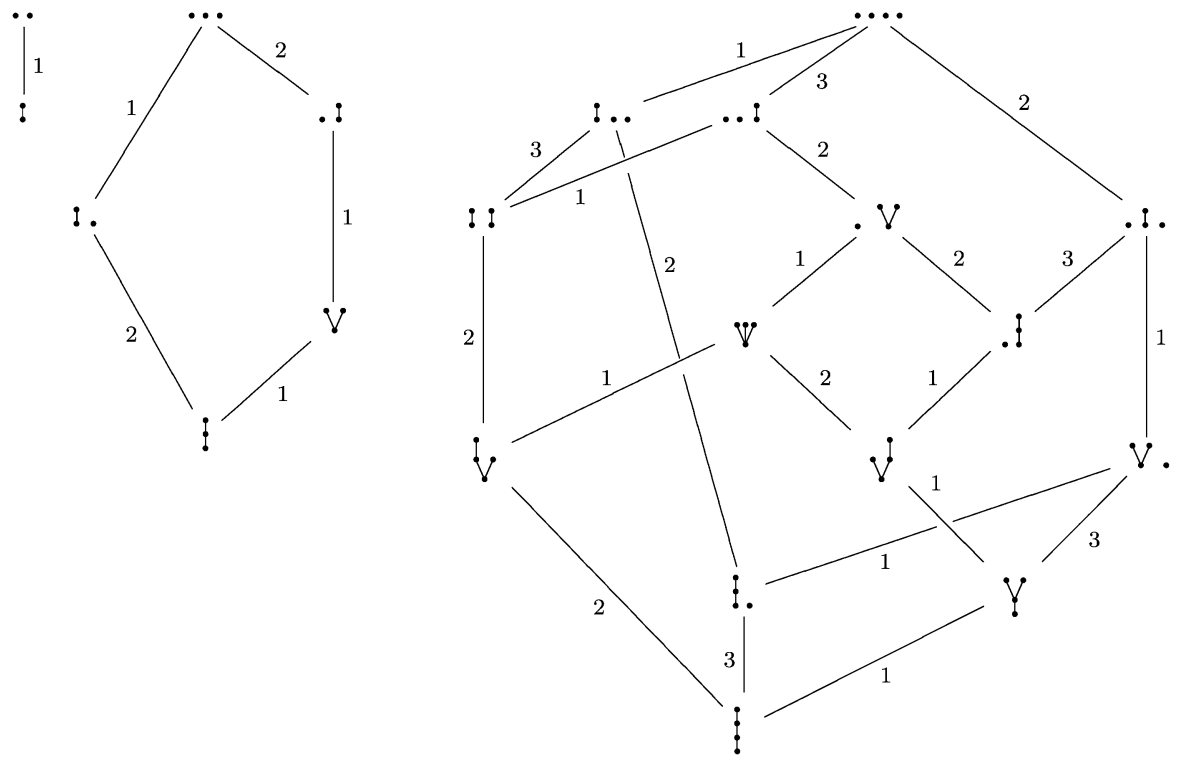

The indices on the edges give the indices of the corresponding admissible transformation. To obtain the Hasse graph of $\geq_{I}$, it is enough to delete the edges whose indices are not in $I$.

\subsection{Application to the product in the dual basis}

The coproduct of a forest can be expressed in terms of the order relations $\leq_{I}$ : 
Proposition 28 Let $F \in \mathbf{F}-\{1\}$. Then:

$$
\Delta(F)=F \otimes 1+1 \otimes F+\sum_{\substack{F_{1}, F_{2} \in \mathbf{F}-\{1\} \\ F \leq\left\{\text { weight }\left(F_{1}\right)\right\}}} F_{1} F_{2}
$$

Proof. By induction on $n=$ weight $(F)$. If $n=1$, then $F=$. and the result is obvious. Suppose the result true at ranks $\leq n-1$. Two cases are possible.

1. $F=F_{1} F_{2}$, and the induction hypothesis holds for $F_{1}$ and $F_{2}$. Then:

$$
\begin{aligned}
& \tilde{\Delta}(F)=\left(F_{1} \otimes 1\right) \tilde{\Delta}\left(F_{2}\right)+\tilde{\Delta}\left(F_{1}\right)\left(1 \otimes F_{2}\right)+F_{1} \otimes F_{2} \\
& =\sum_{\substack{G_{1}, G_{2} \in \mathbf{F}-\{1\} \\
F_{2} \leq\left\{\text { weight }\left(G_{1}\right)\right\}}} F_{1} G_{2} G_{1} \otimes G_{2} \\
& +\sum_{\substack{G_{1}, G_{2} \in \mathbf{F}-\{1\} \\
F_{1} \leq\left\{\text { weight }\left(G_{1}\right)\right\}}} G_{1} G_{2} \otimes F_{2} G_{2}+F_{1} \otimes F_{2} \\
& =\sum_{\substack{G_{1}, G_{2} \in \mathbf{F}-\{1\} \\
\text { weight }\left(G_{1}\right)>\text { weight }\left(F_{1}\right) \\
F \leq\left\{\text { weight }\left(G_{1}\right)\right\}}} G_{1} \otimes G_{2} \otimes G_{2}+\sum_{\begin{array}{c}
G_{1}, G_{2} \in \mathbf{F}-\{1\} \\
\text { weight }\left(G_{1}\right)<\text { weight }\left(F_{1}\right) \\
F \leq\left\{\text { weight }\left(G_{1}\right)\right\} G_{1} G_{2}
\end{array}} G_{1} \otimes G_{2} \\
& +\sum_{\substack{G_{1}, G_{2} \in \mathbf{F}-\{1\} \\
\text { weight }\left(G_{1}\right)=\text { weight }\left(F_{1}\right) \\
F \leq\left\{\text { weight }\left(G_{1}\right)\right\}}} G_{1} G_{2} \otimes G_{2} .
\end{aligned}
$$

So the result is true for $F$.

2. $F=B^{+}(G)$, and the induction hypothesis holds for $G$. Then:

$$
\begin{aligned}
& \tilde{\Delta}(F)=G \otimes \cdot+\left(I d \otimes B^{+}\right) \circ \tilde{\Delta}(G) \\
& =G \otimes \cdot+\sum_{\substack{F_{1}, F_{2} \in \mathbf{F}-\{1\} \\
G \leq\left\{\text { weight }\left(F_{1}\right)\right\}}} F_{1} F_{2} \\
& =\sum_{\begin{array}{c}
F_{1}, F_{2} \in \mathbf{F}-\{1\} \\
\text { weight }\left(F_{2}\right)=1 \\
F \leq\left\{\text { weight }\left(F_{1}\right)\right\}
\end{array} F_{1} F_{2}} F_{1} \otimes F_{2}+\sum_{\begin{array}{c}
F_{1}, F_{2} \in \mathbf{F}-\{1\} \\
\text { weight }\left(F_{2}\right)>1 \\
\left.F \leq \text { weight }\left(F_{1}\right)\right\}
\end{array} F_{1} F_{2}} F_{1} \otimes F_{2} .
\end{aligned}
$$

So the result is true for $F$.

Dually:

Corollary 29 Let $F_{1}, F_{2} \in \mathbf{F}-\{1\}$. Then $f_{F_{2}} f_{F_{1}}=\sum_{\substack{G \in \mathbf{F} \\ G \leq\left\{\text { weight }\left(F_{1}\right)\right\}} F_{1} F_{2}} f_{G}$. 
Proof. We put $f_{F_{2}} f_{F_{1}}=\sum_{G \in \mathbf{F}} a_{G} f_{G}$. Then:

$$
\begin{aligned}
a_{G} & =\left\langle f_{F_{2}} f_{F_{1}}, G\right\rangle \\
& =\left\langle f_{F_{1}} \otimes f_{F_{2}}, \Delta(G)\right\rangle \\
& =\left\langle f_{F_{1}} \otimes f_{F_{2}}, G \otimes 1+1 \otimes G+\sum_{\substack{G_{1}, G_{2} \in \mathbf{F}-\{1\} \\
G_{\left\{\text {weight }\left(G_{1}\right)\right\}} G_{1} G_{2}}} G_{1} \otimes G_{2}\right\rangle \\
& =\sum_{\substack{G_{1}, G_{2} \in \mathbf{F}-\{1\} \\
G_{\left\{\text {weight }\left(G_{1}\right)\right\}} G_{1} G_{2}}} \delta_{F_{1}, G_{1}} \delta_{F_{2}, G_{2}} .
\end{aligned}
$$

So $a_{G}=1$ if $G \leq\left\{\right.$ weight $\left.\left(F_{1}\right)\right\} F_{1} F_{2}$, and 0 if not.

\section{Examples.}

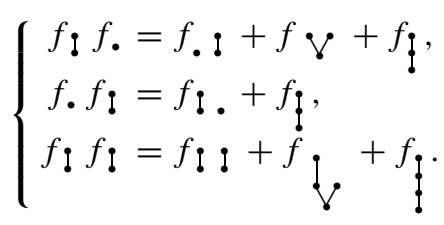

The following result can be proved by induction on $n$ :

Corollary 30 Let $F_{1}, \ldots, F_{n} \in \mathbf{F}-\{1\}$. We define:

$$
I=\left\{\text { weight }\left(F_{1}\right), \text { weight }\left(F_{1} F_{2}\right), \ldots, \text { weight }\left(F_{1} \ldots F_{n-1}\right)\right\} .
$$

Then:

$$
f_{F_{n}} \ldots f_{F_{1}}=\sum_{\substack{G \in \mathbf{F} \\ G \leq I F_{1} \ldots F_{n}}} f_{G} .
$$

For example, for $F_{1}=\ldots=F_{n}=$. , then $I=\{1, \ldots, n-1\}$, so $\geq_{I}=\geq$ on $\mathbf{F}(n)$. By corollary 23, for all $F \in \mathbf{F}(n), F \leq \cdot{ }^{n}$, so $\cdot^{n}$ is the greatest element of $\mathbf{F}(n)$ (it is of course also possible to prove directly this result).

3.3 Isomorphism of $(\mathbf{F}, \leq)$ with the Tamari poset

Let $\mathbf{T}_{b}$ be the set of planar binary trees and, for all $n \in \mathbb{N}$, let $\mathbf{T}_{b}(n)$ be the set of planar binary trees with $n$ internal vertices (and $n+1$ leaves). For example:

$$
\begin{aligned}
& \mathbf{T}_{b}(0)=\{1\}, \mathbf{T}_{b}(1)=\{Y\}, \mathbf{T}_{b}(2)=\{Y, Y\} \\
& \mathbf{T}_{b}(3)=\{Y, Y, Y, Y, Y Y \\
& Y, Y
\end{aligned}
$$


The set $\mathbf{T}_{b}$ is given the Tamari order (see $[4,5,18,23]$ ). Let us recall that it is the order generated by the local Tamari transformation:

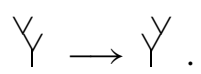

Let $t_{1}, t_{2}$ be two planar binary trees. We denote by $t_{1} \vee t_{2}$ the planar binary tree obtained by grafting $t_{1}$ and $t_{2}$ on a common root. For example:

$$
\text { । } \vee Y=Y, Y \vee Y=Y Y
$$

Note that every planar binary tree different from । can be uniquely written $t^{l} \vee t^{r}$, where $t^{l}$ and $t^{r}$ are two planar binary trees.

We define a map $\eta: \mathbf{T}_{b} \longrightarrow \mathbf{F}$ by induction on the number of internal vertices by:

$$
\eta:\left\{\begin{aligned}
\mathbf{T}_{b} & \longrightarrow \mathbf{F} \\
\mid & \longrightarrow 1 \\
t_{1} \vee t_{2} & \longrightarrow B^{+}\left(\eta\left(t_{1}\right)\right) \eta\left(t_{2}\right) .
\end{aligned}\right.
$$

It is not difficult to prove that $\eta$ is bijective, with inverse given by:

$$
\eta^{-1}:\left\{\begin{aligned}
\mathbf{F} & \longrightarrow \mathbf{T}_{b} \\
1 & \longrightarrow \text { । } \\
B^{+}\left(F_{1}\right) F_{2} & \longrightarrow \eta^{-1}\left(F_{1}\right) \vee \eta^{-1}\left(F_{2}\right) .
\end{aligned}\right.
$$

Moreover, $\eta$ induces a bijection: $\eta: \mathbf{T}_{b}(n) \longrightarrow \mathbf{F}(n)$, for all $n \in \mathbb{N}$.

\section{Examples.}

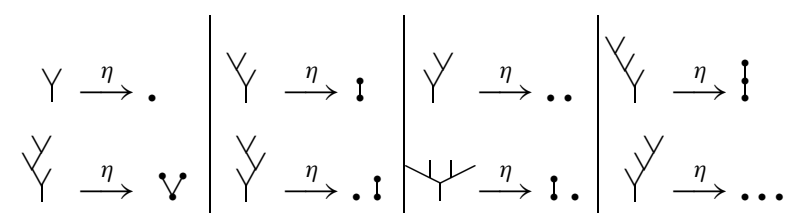

The aim of this subsection is to show the following theorem:

Theorem 31 Let $t_{1}, t_{2} \in \mathbf{T}_{b}$. The following equivalence holds:

$$
\left(t_{1} \leq t_{2}\right) \Longleftrightarrow\left(\eta\left(t_{1}\right) \leq \eta\left(t_{2}\right)\right)
$$

In other terms, $\eta$ is a poset isomorphism.

Let us define $\backslash: \mathbf{T}_{b} \times \mathbf{T}_{b} \longrightarrow \mathbf{T}_{b}$ in the following way (see [18]): $t_{1} \backslash t_{2}$ is the grafting of $t_{2}$ on the leave of $t_{1}$ at most on the right. Note that $\backslash$ is an associative operation, and every $t \in \mathbf{T}_{b}$ can be uniquely decomposed in the form $t=\left(t_{1} \vee \mathrm{I}\right) \backslash \ldots \backslash\left(t_{n} \vee \mathrm{I}\right)$, 
with $n \in \mathbb{N}$ and $t_{1}, \ldots, t_{n} \in \mathbf{T}_{b}$.

\section{Examples.}

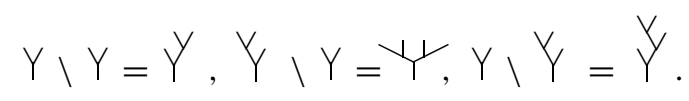

Lemma 32 The Tamari order on $\mathbf{T}_{b}$ is the least fine partial order satisfying:

1. for all $t_{1}, t_{2}, t_{3} \in \mathbf{T}_{b},\left(t_{1} \leq t_{2}\right) \Longrightarrow\left\{\begin{array}{l}t_{1} \vee 1 \leq t_{2} \vee \text { ।, } \\ t_{1} \backslash t_{3} \leq t_{2} \backslash t_{3}, \\ t_{3} \backslash t_{1} \leq t_{3} \backslash t_{2}\end{array}\right.$

2. for all $t_{1}, t_{2} \in \mathbf{T}_{b},\left(t_{1} \vee t_{2}\right) \vee 1 \leq t_{1} \vee\left(t_{2} \vee ।\right)$.

Proof. Let $\sqsubseteq$ be the least fine partial order satisfying the assertions of lemma 32 . Clearly, the Tamari order $\leq$ satisfies these conditions, so for all $t_{1}, t_{2} \in \mathbf{T}_{b},\left(t_{1} \sqsubseteq\right.$ $\left.t_{2}\right) \Longrightarrow\left(t_{1} \leq t_{2}\right)$.

Let $t_{1}, t_{2} \in \mathbf{T}_{b}$, such that $t_{1} \leq t_{2}$. Let us show that $t_{1} \sqsubseteq t_{2}$. First, remark that $t_{1}$ and $t_{2}$ have the same number $n$ of internal vertices. Let us proceed by induction on $n$. If $n=0$, then $t_{1}=t_{2}=1$ and the result is obvious. If $n \geq 1$, we can suppose that $t_{2}$ is

obtained from $t_{1}$ by a single Tamari operation $Y \rightarrow Y$. Let us put $t_{1}=t_{1}^{\prime} \vee t_{1}^{\prime \prime}$ and $t_{2}=t_{2}^{\prime} \vee t_{2}^{\prime \prime}$. Three cases are possible.

1. If the operation holds on a vertex of $t_{1}^{\prime}$, then $t_{1}^{\prime} \leq t_{2}^{\prime}$ and $t_{1}^{\prime \prime}=t_{2}^{\prime \prime}$. By induction hypothesis, $t_{1}^{\prime} \leq t_{2}^{\prime}$. So, $t_{1}^{\prime} \vee \mathrm{I} \sqsubseteq t_{2}^{\prime} \vee \mathrm{I}$ and $t_{1}=\left(t_{1}^{\prime} \vee \mathrm{I}\right) \backslash t_{1}^{\prime \prime} \sqsubseteq\left(t_{2}^{\prime} \vee \mathrm{I}\right) \backslash t_{1}^{\prime \prime}=t_{2}$.

2. If the operation holds on a vertex of $t_{1}^{\prime \prime}$, then $t_{1}^{\prime}=t_{2}^{\prime}$ and $t_{1}^{\prime \prime} \sqsubseteq t_{2}^{\prime \prime}$. By induction hypothesis, $t_{1}=\left(t_{1}^{\prime} \vee \mathrm{I}\right) \backslash t_{1}^{\prime \prime} \sqsubseteq\left(t_{2}^{\prime} \vee \mathrm{I}\right) \backslash t_{1}^{\prime \prime}=t_{2}$.

3. If the operation holds on the root of $t_{1}$, let us put $t_{1}=\left(t_{1}^{\prime} \vee t_{1}^{\prime \prime}\right) \vee t_{1}^{\prime \prime \prime}$. Then $t_{2}=$ $t_{1}^{\prime} \vee\left(t_{1}^{\prime \prime} \vee t_{1}^{\prime \prime \prime}\right)$. Hence, $\left(t_{1}^{\prime} \vee t_{1}^{\prime \prime}\right) \vee । \sqsubseteq t_{1}^{\prime} \vee\left(t_{1}^{\prime \prime} \vee \mathrm{I}\right)$ and $t_{1}=\left(\left(t_{1}^{\prime} \vee t_{1}^{\prime \prime}\right) \vee \mathrm{I}\right) \backslash t_{1}^{\prime \prime \prime} \sqsubseteq$ $\left(t_{1}^{\prime} \vee\left(t_{1}^{\prime \prime} \vee \mid\right)\right) \backslash t_{1}^{\prime \prime \prime}=t_{2}$

Lemma 33 The partial order $\leq$ on $\mathbf{F}$ is the least fine partial order satisfying:

1. For all $F_{1}, F_{2}, F_{3} \in \mathbf{F},\left(F_{1} \leq F_{2}\right) \Longrightarrow\left\{\begin{aligned} B^{+}\left(F_{1}\right) & \leq B^{+}\left(F_{2}\right) \text {, } \\ F_{1} F_{3} & \leq F_{2} F_{3}, \\ F_{3} F_{1} & \leq F_{3} F_{2} .\end{aligned}\right.$

2. For all $\left.F_{1}, F_{2} \in \mathbf{F}, B^{+}\left(B^{+}\left(F_{1}\right) F_{2}\right)\right) \leq B^{+}\left(F_{1}\right) B^{+}\left(F_{2}\right)$.

Proof. Let $\sqsubseteq$ be the least fine partial order on $\mathbf{F}$ satisfying the assertions of lemma 33 . As the order $\leq$ clearly satisfies these conditions, for all $F_{1}, F_{2} \in \mathbf{F},\left(F_{1} \sqsubseteq F_{2}\right) \Longrightarrow$ $\left(F_{1} \leq F_{2}\right)$.

Let $F_{1}, F_{2} \in \mathbf{F}$, such that $F_{1} \leq F_{2}$. Let us show $F_{1} \sqsubseteq F_{2}$. Necessarily, $F_{1}$ and $F_{2}$ have the same weight $n$. Let us proceed by induction on $n$. If $n=0$, then $F_{1}=F_{2}=1$ and the result is obvious. Suppose $n \geq 1$. We can suppose that $F_{2}$ is obtained from $F_{1}$ by a single elementary operation. Three cases are possible.

1. $F_{1}=B^{+}\left(G_{1}\right)$ and the operation holds on $G_{1}$. Then $F_{2}=B^{+}\left(G_{2}\right)$, with $G_{1} \leq G_{2}$.

By induction hypothesis, $G_{1} \sqsubseteq G_{2}$. So, $F_{1}=B^{+}\left(G_{1}\right) \sqsubseteq B^{+}\left(G_{2}\right)=F_{2}$. 
2. $F_{1}=B^{+}\left(G_{1}\right)$ and the transformation is of second kind. We then put $G_{1}=t_{1} G_{1}^{\prime}$ with $t_{1} \in \mathbf{T}$. Then $F_{2}=t_{1} B^{+}\left(G_{1}^{\prime}\right)$. By property 2 of $\sqsubseteq, F_{1} \sqsubseteq F_{2}$.

3. $F_{1}=t_{1} \ldots t_{k}, k \geq 2$, and the transformation holds on $t_{i}$. We can then write $F_{2}=$ $t_{1} \ldots t_{i-1} G_{i} t_{i+1} \ldots t_{k}$, with $t_{i} \leq G_{i}$. By induction hypothesis, $t_{i} \sqsubseteq G_{i}$. Hence, $t_{1} \ldots t_{i} \sqsubseteq t_{1} \ldots t_{i-1} G_{i}$ and $F_{1}=t_{1} \ldots t_{k} \sqsubseteq t_{1} \ldots t_{i-1} G_{i} t_{i+1} \ldots t_{k}=F_{2}$.

Lemma $34 \eta$ satisfies the following assertions:

1. for all $t \in \mathbf{T}_{b}, \eta(t \vee$ । $)=B^{+}(\eta(t))$.

2. for all $t_{1}, t_{2} \in \mathbf{T}_{b}, \eta\left(t_{1} \backslash t_{2}\right)=\eta\left(t_{1}\right) \eta\left(t_{2}\right)$.

\section{Proof.}

1. Indeed, $\eta(t \vee \mid)=B^{+}(\eta(t)) \eta(1)=B^{+}(\eta(t)) 1=B^{+}(\eta(t))$.

2. Let us put $t_{1}=\left(s_{1} \vee ।\right) \backslash \ldots \backslash\left(s_{k} \vee ।\right)$. We proceed by induction on $k$. If $k=0$, then $t_{1}=\mid$ and $\eta\left(t_{1}\right)=1$, so $\eta\left(t_{1} \backslash t_{2}\right)=\eta\left(t_{2}\right)=\eta\left(t_{1}\right) \eta\left(t_{2}\right)$. If $k=1$, then $t_{1} \backslash t_{2}=$ $s_{1} \vee t_{2}$ and $\eta\left(t_{1}\right)=B^{+}\left(\eta\left(s_{1}\right)\right)$ by the first point. So, $\eta\left(t_{1} \backslash t_{2}\right)=B^{+}\left(\eta\left(s_{1}\right)\right) \eta\left(t_{2}\right)=$ $\eta\left(t_{1}\right) \eta\left(t_{2}\right)$. Let us suppose the result at rank $k-1$.

$$
\begin{aligned}
\eta\left(t_{1} \backslash t_{2}\right) & =\eta\left(\left(s_{1} \vee ।\right) \backslash \ldots \backslash\left(s_{k} \vee ।\right) \backslash t_{2}\right) \\
& =\eta\left(s_{1} \vee ।\right) \eta\left(\left(s_{2} \vee ।\right) \backslash \ldots \backslash\left(s_{k} \vee ।\right) \backslash t_{2}\right) \\
& =\eta\left(s_{1} \vee ।\right) \eta\left(s_{2} \vee ।\right) \ldots \eta\left(s_{k} \vee ।\right) \eta\left(t_{2}\right) \\
& =\eta\left(\left(s_{1} \vee ।\right) \backslash \ldots \backslash\left(s_{k} \vee ।\right)\right) \eta\left(t_{2}\right) \\
& =\eta\left(t_{1}\right) \eta\left(t_{2}\right) .
\end{aligned}
$$

We used the result at rank 1 for the second equality and the result at rank $k-1$ for the third and the fourth ones.

Proof of theorem 31. We define $\preceq$ on $\mathbf{T}_{b}$ by $\left(t_{1} \preceq t_{2}\right) \Longleftrightarrow\left(\eta\left(t_{1}\right) \leq \eta\left(t_{2}\right)\right)$. As $\eta$ is a bijection, this is a partial order on $\mathbf{T}_{b}$. By lemmas 33 and 34, this is the least fine partial order satisfying the conditions of lemma 32 . So it is the Tamari order.

\subsection{An order-reversing automorphism of the poset $\mathbf{F}$}

Proposition 35 We define an involution $m: \mathbf{F} \longrightarrow \mathbf{F}$ by induction on the weight in the following way:

$$
\left\{\begin{aligned}
m(1) & =1, \\
m\left(B^{+}\left(F_{1}\right) F_{2}\right) & =B^{+}\left(m\left(F_{2}\right)\right) m\left(F_{1}\right) \text { for any } F_{1}, F_{2} \in \mathbf{F} .
\end{aligned}\right.
$$

Proof. Clearly, this defines inductively $m(F)$ for all forest $F$ in a unique way. Let us show that $m^{2}(F)=F$ for all forest $F \in \mathbf{F}$ by induction on the weight $n$ of $F$. If $n=0$, the result is obvious. Suppose the result true for all rank $<n$. Let $F \in \mathbf{F}(n)$. We put $F=B^{+}\left(F_{1}\right) F_{2}$. Then the induction hypothesis holds for $F_{1}$. So:

$m \circ m(F)=m\left(B^{+}\left(m\left(F_{2}\right)\right) m\left(F_{1}\right)\right)=B^{+}\left(m \circ m\left(F_{1}\right)\right) m \circ m\left(F_{2}\right)=B^{+}\left(F_{1}\right) F_{2}=F$. 
So $m$ is an involution.

Remark. For all forest $F \in \mathbf{F}, F$ and $m(F)$ have the same weight. So $m$ induces an involution $m: \mathbf{F}(n) \longrightarrow \mathbf{F}(n)$ for all $n \in \mathbb{N}$.

\section{Examples.}

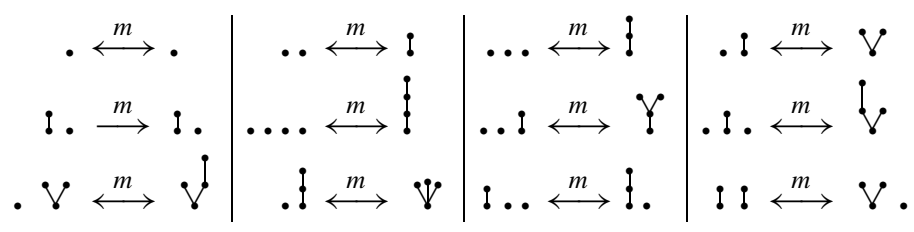

Proposition 36 Let $F, G \in \mathbf{F}$. Then $F \leq G$ if, and only if, $m(G) \leq m(F)$.

Proof. We consider the bijection $m^{\prime}: \mathbf{T}_{b} \longrightarrow \mathbf{T}_{b}$ defined by $m^{\prime}=\eta^{-1} \circ m \circ \eta$. Then $m^{\prime}(1)=1$, and for all $t_{1}, t_{2} \in \mathbf{T}_{b}$ :

$$
\begin{aligned}
m^{\prime}\left(t_{1} \vee t_{2}\right) & =\eta^{-1} \circ m\left(B^{+}\left(\eta\left(t_{1}\right)\right) \eta\left(t_{2}\right)\right) \\
& =\eta^{-1}\left(B^{+}\left(m \circ \eta\left(t_{2}\right)\right) m \circ \eta\left(t_{1}\right)\right) \\
& =\eta^{-1} \circ m \circ \eta\left(t_{2}\right) \vee \eta^{-1} \circ m \circ \eta\left(t_{1}\right) \\
& =m^{\prime}\left(t_{2}\right) \vee m^{\prime}\left(t_{1}\right) .
\end{aligned}
$$

Hence, $m^{\prime}$ is the vertical reflection:

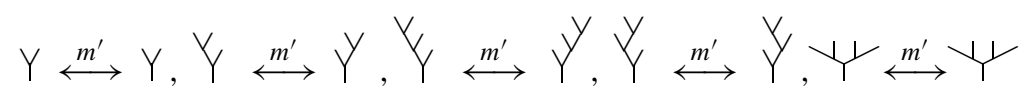

It is obviously an order-reversing automorphism of $\mathbf{T}_{b}$. If $F, G \in \mathbf{F}$, let us put $t_{1}=$ $\eta^{-1}(F)$ and $t_{2}=\eta^{-1}(G)$ :

$$
\begin{aligned}
F \leq G & \Longleftrightarrow \eta\left(t_{1}\right) \leq \eta\left(t_{2}\right) \\
& \Longleftrightarrow t_{1} \leq t_{2} \\
& \Longleftrightarrow m^{\prime}\left(t_{2}\right) \leq m^{\prime}\left(t_{1}\right) \\
& \Longleftrightarrow \eta \circ m^{\prime}\left(t_{2}\right) \leq \eta \circ m^{\prime}\left(t_{1}\right) \\
& \Longleftrightarrow \eta \circ m^{\prime} \circ \eta^{-1}(G) \leq \eta \circ m^{\prime} \circ \eta^{-1}(F) \\
& \Longleftrightarrow m(G) \leq m(F) .
\end{aligned}
$$

Hence, $m$ is order-reversing.

Remark. So $m\left(\cdot^{n}\right)=\left(B^{+}\right)^{n}(1)$ (ladder of weight $n$ ) is the smallest element of $\mathbf{F}(n)$. 
3.5 Link between the pairing and the partial order

Theorem 37 Let $F, G \in \mathbf{F}$. The following assertions are equivalent:

1. $\langle F, G\rangle \neq 0$.

2. $\langle F, G\rangle=1$.

3. $m(G) \leq F$.

\section{Proof.}

$1 \Longrightarrow 3$. Let us suppose that $\langle F, G\rangle \neq 0$ and let us show that $F \geq m(G)$. We proceed by induction on weight $(F)=$ weight $(G)=n$. If $n=0$, then $F=$ $G=1$ and $F \geq m(G)$. We now suppose the result at all rank $<n$. We put $G=B^{+}\left(G_{1}\right) G_{2}$. Then the induction hypothesis holds for $G_{1}$ and $G_{2}$. Moreover, $m(G)=B^{+}\left(m\left(G_{2}\right)\right) m\left(G_{1}\right)$. Let $c$ be the unique totally admissible cut of $F$ such that $P^{c}(F)$ and $G_{2}$ have the same weight. Then, by homogeneity of $\langle-,-\rangle$ :

$$
\begin{aligned}
\langle F, G\rangle & =\left\langle F, B\left(G_{1}\right) G_{2}\right\rangle=\left\langle\Delta(F), G_{2} \otimes B\left(G_{1}\right)\right\rangle \\
& =\left\langle P^{c}(F) \otimes R^{c}(F), G_{2} \otimes B\left(G_{1}\right)\right\rangle=1 .
\end{aligned}
$$

As $\langle F, G\rangle \neq 0$, then $\left\langle P^{c}(F), G_{2}\right\rangle$ and $\left\langle R^{c}(F), B\left(G_{1}\right)\right\rangle$ are non zero. By the induction hypothesis, $P^{c}(F) \geq m\left(G_{2}\right)$ and $R^{c}(F) \geq m\left(B\left(G_{1}\right)\right)=\cdot m\left(G_{1}\right)$. As $c$ is a totally admissible cut, we easily deduce that $F \geq B^{+}\left(m\left(G_{2}\right)\right) m\left(G_{1}\right)=m(G)$.

$3 \Longrightarrow 2$. Let us suppose that $m(G) \leq F$ and let us show that $\langle F, G\rangle=1$. We proceed by induction on the common weight $n$ of $G$ and $F$. If $n=0$, then $F=G=1$ and $\langle F, G\rangle=1$. We now suppose the result at all rank $<n$. We put $G=B^{+}\left(G_{1}\right) G_{2}$, with $G_{1}, G_{2} \in \mathbf{F}$. Then $m(G)=B^{+}\left(m\left(G_{2}\right)\right) m\left(G_{1}\right)$ and $F \geq B^{+}\left(m\left(G_{2}\right)\right) m\left(G_{1}\right)$. Let $c$ be the unique totally admissible cut of $F$ such that $P^{c}(F)$ and $G_{2}$ have the same weight. By definition of $\geq, P^{c}(F) \geq m\left(G_{2}\right)$ and $R^{c}(F) \geq \cdot m\left(G_{1}\right)=m\left(B^{+}\left(G_{1}\right)\right)$. Hence, $\left\langle P^{c}(F), G_{2}\right\rangle=\left\langle R^{c}(F), B^{+}\left(G_{1}\right)\right\rangle=1$. As $\langle-,-\rangle$ is homogeneous:

$$
\begin{aligned}
\langle F, G\rangle & =\left\langle F, B^{+}\left(G_{1}\right) G_{2}\right\rangle=\left\langle\Delta_{0}(F), G_{2} \otimes B^{+}\left(G_{1}\right)\right\rangle \\
& =\left\langle P^{c}(F) \otimes R^{c}(F), G_{2} \otimes B^{+}\left(G_{1}\right)\right\rangle=1 .
\end{aligned}
$$

$2 \Longrightarrow 1$. Obvious.

Examples. Values of the pairing $\langle-,-\rangle$ for forests of weight $\leq 4$ :
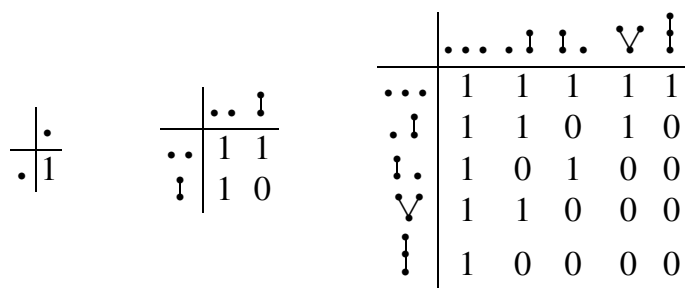


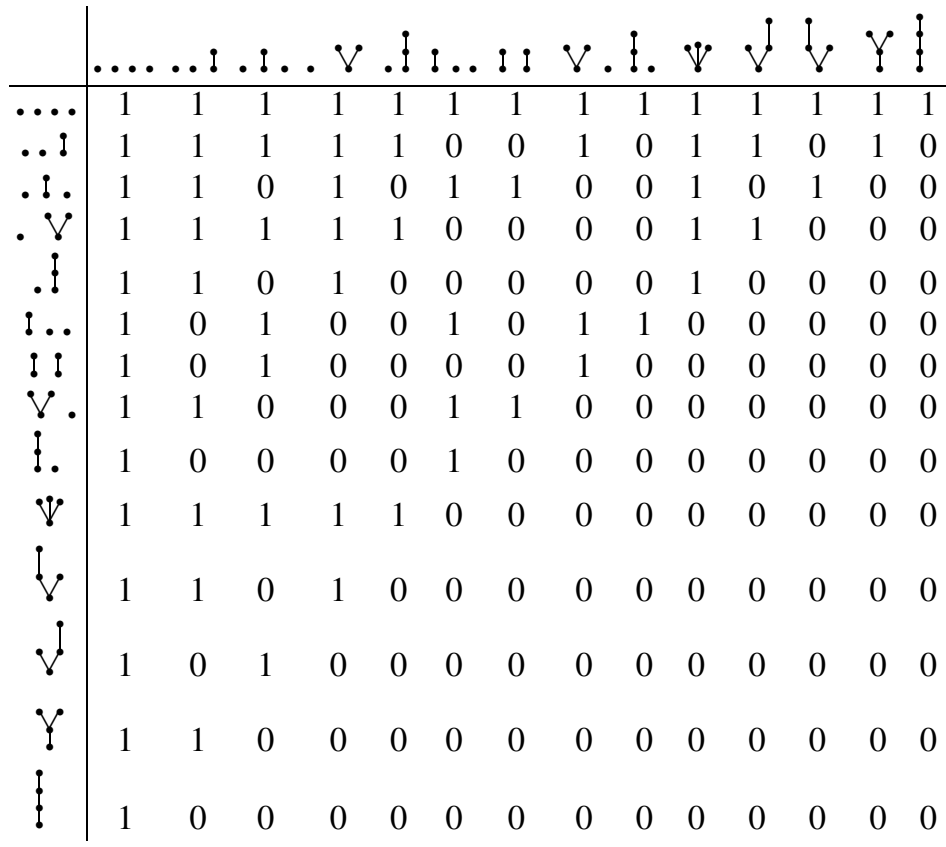

As a corollary, we can give another (shorter) proof of the symmetry of $\langle-,-\rangle$ : if $F, G \in \mathbf{F}$,

$$
\begin{aligned}
(\langle F, G\rangle=1) & \Longleftrightarrow(F \geq m(G)) \Longleftrightarrow\left(m^{2}(F) \geq m(G)\right) \Longleftrightarrow(G \geq m(F)) \\
& \Longleftrightarrow(\langle G, F\rangle=1) .
\end{aligned}
$$

Corollary 38 Let $F \in \mathbf{F}(n)$. Then $F=\sum_{\substack{G \in \mathbf{F}(n) \\ F \geq m(G)}} f_{G}=\sum_{\substack{G \in \mathbf{F}(n) \\ F \geq G}} f_{m(G)}$.

Proof. We put $F=\sum_{G \in \mathbf{F}(n)} a_{G, F} f_{G}$. Then $a_{G, F}=\langle F, G\rangle$, which implies this corollary.

Let $\mu: \mathbf{F}(n)^{2} \longrightarrow K$ be the Möbius function of the poset $\mathbf{F}(n)$, that is to say (see [22]):

1. $\mu(F, G)=0$ if $F \not \leq G$.

2. $\sum_{\substack{G \in \mathbf{F}(n) \\ F \leq G \leq H}} \mu(F, G)=\delta_{F, H}$ if $F \leq H$.

Immediately, by [22]: 
Corollary 39 Let $F \in \mathbf{F}(n)$. Then $f_{F}=\sum_{\substack{G \in \mathbf{F}(n) \\ G \leq m(F)}} \mu(G, m(F)) G$.

\section{Examples.}

\begin{tabular}{|c|c|}
\hline 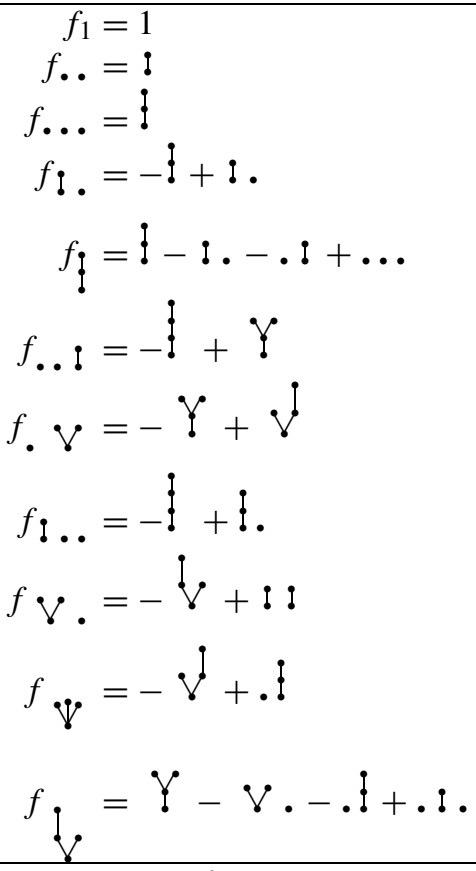 & 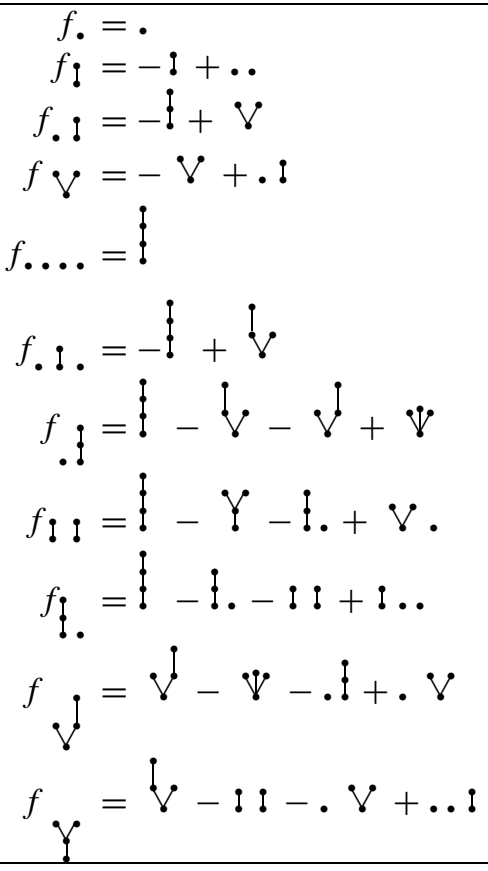 \\
\hline$:=-!+\vdots+! !-!$ & \\
\hline
\end{tabular}

\section{References}

1. Abe, E.: Hopf algebras. Cambridge Tracts in Mathematics, vol. 74. Cambridge University Press, Cambridge-New York (1980)

2. Aguiar, M.: Infinitesimal Hopf algebras. Contemp. Math. 267, 1-29 (2000)

3. Aguiar, M.: Infinitesimal bialgebras, pre-Lie and dendriform algebras. Lecture Notes in Pure and Appl. Math., vol. 237. Dekker, New York (2004)

4. Aguiar, M., Sottile, F.: Structure of the Malvenuto-Reutenauer Hopf algebra of permutations. Adv. Math. 191(2), 225-275 (2005). math.CO/0203282

5. Aguiar, M., Sottile, F.: Structure of the Loday-Ronco Hopf algebra of trees. J. Algebra 295(2), 473511 (2006). math.CO/0409022

6. Connes, A., Kreimer, D.: Hopf algebras, Renormalization and Noncommutative geometry. Comm. Math. Phys 199(1), 203-242 (1998). hep-th/9808042

7. Connes, A., Kreimer, D.: Renormalization in quantum field theory and the Riemann-Hilbert problem I. The Hopf algebra of graphs and the main theorem. Comm. Math. Phys. 210(1), 249-273 (2000). hep-th/9912092

8. Connes, A., Kreimer, D.: Renormalization in quantum field theory and the Riemann-Hilbert problem. II. The $\beta$-function, diffeomorphisms and the renormalization group. Comm. Math. Phys. 216(1), 215241 (2001). hep-th/0003188 
9. Doi, Y.: Homological coalgebra. J. Math. Soc. Japan 33(1), 31-50 (1981)

10. Foissy, L.: Finite-dimensional comodules over the Hopf algebra of rooted trees. J. Algebra 255(1), 85-120 (2002). math.QA/0105210

11. Foissy, L.: Les algèbres de Hopf des arbres enracinés, I. Bull. Sci. Math. 126, 193-239 (2002)

12. Foissy, L.: Quantifications des algèbres de Hopf d'arbres plans décorés et lien avec les groupes quantiques. Bull. Sci. Math. 127(6), 505-548 (2003)

13. Grossman, R., Larson, R.G.: Hopf-algebraic structure of families of trees. J. Algebra 126(1), 184-210 (1989)

14. Holtkamp, R.: Comparison of Hopf Algebras on Trees. Arch. Math. (Basel) 80(4), 368-383 (2003)

15. Kreimer, D.: On the Hopf algebra structure of pertubative quantum field theories. Adv. Theor. Math. Phys. 2(2), 303-334 (1998). q-alg/9707029

16. Kreimer, D.: On Overlapping Divergences. Comm. Math. Phys. 204(3), 669-689 (1999). hep-th/9810022

17. Kreimer, D.: Combinatorics of (pertubative) Quantum Field Theory. Phys. Rep. 4-6, 387-424 (2002). hep-th/0010059

18. Loday, J.-L., Ronco, M.O.: Hopf algebra of the planar binary trees. Adv. Math. 139(2), 293-309 (1998)

19. Loday, J.-L., Ronco, M.O.: On the structure of cofree hopf algebras. J. Reine Angew. Math. 592, 123-155 (2006)

20. Moerdijk, I.: On the Connes-Kreimer construction of Hopf algebras. Contemp. Math. 271, 311-321 (2001). math-ph/9907010

21. Schmitt, W.R.: Incidence Hopf algebras. J. Pure Appl. Algebra 96(3), 299-330 (1994)

22. Stanley, R.P.: Enumerative combinatorics. Vol. 1. Cambridge Studies in Advanced Mathematics, vol. 49. Cambridge University Press, Cambridge (1997)

23. Stanley, R.P.: Enumerative combinatorics. Vol. 2. Cambridge Studies in Advanced Mathematics, vol. 62. Cambridge University Press, Cambridge (1999)

24. Sweedler, M.E.: Hopf algebras. Mathematics Lecture Note Series. Benjamin, New York (1969)

25. Takeuchi, M.: Survey of braided Hopf algebras. Contemp. Math., vol. 267. Amer. Math. Soc., Providence (2000) 